\title{
Article
}

\section{Some integral inequalities for co-ordinated harmonically convex functions via fractional integrals}

\author{
Naila Mehreen ${ }^{1, *}$ and Matloob Anwar ${ }^{1}$ \\ 1 School of Natural Sciences, National University of Sciences and Technology, H-12 Islamabad, Pakistan. \\ * Correspondence: nailamehreen@gmail.com \\ Received: 20 June 2020; Accepted: 30 November 2020; Published: 16 December 2020.
}

\begin{abstract}
In this paper, we find some Hermite-Hadamard type inequalities for co-ordinated harmonically convex functions via fractional integrals.
\end{abstract}

Keywords: Hermite-Hadamard inequalities, Riemann-Liouville fractional integral, co-ordinated convex functions, co-ordinated harmonically convex functions.

\section{Introduction and Preliminaries}

F

or a convex mapping $\Pi: I \rightarrow \mathbb{R}$ on a real interval, for all $f_{1}, f_{2} \in I$ and $t \in[0,1]$, the inequality

$$
\prod\left(\frac{f_{1}+f_{2}}{2}\right) \leq \frac{1}{f_{2}-f_{1}} \int_{f_{1}}^{f_{2}} \prod(u) d u \leq \frac{\prod\left(f_{1}\right)+\prod\left(f_{2}\right)}{2},
$$

is known as the Hermite-Hadamard inequality [1]. The inequality (1) has been established for several generalized convex functions [2-9]. Dragomir [10] and Sarikaya [11] calculated Hermite-Hadamard inequality for co-ordinated convex functions. They define co-ordinated convex function as:

Definition 1. [10] A function $\Pi: \Delta=\left[f_{1}, f_{2}\right] \times\left[g_{1}, g_{2}\right] \subseteq \mathbb{R}^{2} \rightarrow \mathbb{R}$ is called co-ordinate convex on $\Delta$ with $f_{1}<f_{2}$ and $g_{1}<g_{2}$, if the partial functions

$$
\prod_{y}:\left[f_{1}, f_{2}\right] \rightarrow \mathbb{R}, \prod_{y}(u)=\Pi(u, y), \text { and } \prod_{x}:\left[g_{1}, g_{2}\right] \rightarrow \mathbb{R}, \prod_{x}(v)=\Pi(x, v),
$$

are convex for all $x \in\left[f_{1}, f_{2}\right]$ and $y \in\left[g_{1}, g_{2}\right]$.

Sarikaya [11] define the co-ordinated convex function as:

Definition 2. [11] A function $\Pi: \Delta=\left[f_{1}, f_{2}\right] \times\left[g_{1}, g_{2}\right] \subseteq \mathbb{R}^{2} \rightarrow \mathbb{R}$ is called coordinate convex on $\Delta$ with $f_{1}<f_{2}$ and $g_{1}<g_{2}$, if

$$
\begin{aligned}
& \prod\left(t_{1} x+\left(1-t_{1}\right) z, t_{2} y+\left(1-t_{2}\right) w\right) \\
& \leq t_{1} t_{2} \prod(x, y)+t_{1}\left(1-t_{2}\right) \prod(x, w)+\left(1-t_{1}\right) t_{2} \prod(z, y)+\left(1-t_{1}\right)\left(1-t_{2}\right) \prod(z, w),
\end{aligned}
$$

holds for all $t_{1}, t_{2} \in[0,1]$ and $(x, y),(z, w) \in \Delta$.

Every convex function is co-ordinated convex but not conversely [10].

Theorem 3. [10] Let $\Pi: \Delta=\left[f_{1}, f_{2}\right] \times\left[g_{1}, g_{2}\right] \subseteq \mathbb{R}^{2} \rightarrow \mathbb{R}$ be convex on $\Delta$ with $f_{1}<f_{2}$ and $g_{1}<g_{2}$. Then

$$
\begin{aligned}
\Pi\left(\frac{f_{1}+f_{2}}{2}, \frac{g_{1}+g_{2}}{2}\right) & \leq \frac{1}{2}\left[\frac{1}{f_{2}-f_{1}} \int_{f_{1}}^{f_{2}} \Pi\left(x, \frac{g_{1}+g_{2}}{2}\right) d x+\frac{1}{g_{2}-g_{1}} \int_{g_{1}}^{g_{2}} \prod\left(\frac{f_{1}+f_{2}}{2}, y\right) d y\right] \\
& \leq \frac{1}{\left(f_{2}-f_{1}\right)\left(g_{2}-g_{1}\right)} \int_{g_{1}}^{g_{2}} \int_{f_{1}}^{f_{2}} \prod(x, y) d x d y
\end{aligned}
$$




$$
\begin{aligned}
\leq & \frac{1}{4}\left[\frac{1}{f_{2}-f_{1}} \int_{f_{1}}^{f_{2}} \prod\left(x, g_{1}\right) d x+\frac{1}{f_{2}-f_{1}} \int_{f_{1}}^{f_{2}} \prod(x, d) d x\right. \\
& \left.+\frac{1}{g_{2}-g_{1}} \int_{g_{1}}^{g_{2}} \prod\left(f_{1}, y\right) d y+\frac{1}{g_{2}-g_{1}} \int_{g_{1}}^{g_{2}} \prod\left(f_{2}, y\right) d y\right] \\
\leq & \frac{\prod\left(f_{1}, g_{2}\right)+\prod\left(f_{1}, g_{2}\right)+\prod\left(f_{2}, g_{1}\right)+\prod\left(f_{2}, g_{2}\right)}{4} .
\end{aligned}
$$

Definition 4. [12] A function $\Pi: \Delta=\left[f_{1}, f_{2}\right] \times\left[g_{1}, g_{2}\right] \subseteq \mathbb{R}^{2} \rightarrow \mathbb{R}$ is called harmonically convex on $\Delta$ with $f_{1}<f_{2}$ and $g_{1}<g_{2}$, if

$$
\prod\left(\frac{x z}{t_{1} x+\left(1-t_{1}\right) z}, \frac{y w}{t_{2} y+\left(1-t_{2}\right) w}\right) \leq t_{1} t_{2} \prod(x, y)+\left(1-t_{1}\right)\left(1-t_{2}\right) \prod(z, w),
$$

holds for all $t_{1}, t_{2} \in[0,1]$ and $(x, y),(z, w) \in \Delta$.

Definition 5. [12] A function $\Pi: \Delta=\left[f_{1}, f_{2}\right] \times\left[g_{1}, g_{2}\right] \subseteq(0, \infty) \times(0, \infty) \rightarrow \mathbb{R}$ is called coordinated harmonically convex on $\Delta$ with $f_{1}<f_{2}$ and $g_{1}<g_{2}$, if

$$
\begin{aligned}
& \prod\left(\frac{x z}{t_{1} x+\left(1-t_{1}\right) z}, \frac{y w}{t_{2} y+\left(1-t_{2}\right) w}\right) \\
& \leq t_{1} t_{2} \prod(x, y)+t_{1}\left(1-t_{2}\right) \prod(x, w)+\left(1-t_{1}\right) t_{2} \prod(z, y)+\left(1-t_{1}\right)\left(1-t_{2}\right) \prod(z, w),
\end{aligned}
$$

holds for all $t_{1}, t_{2} \in[0,1]$ and $(x, y),(z, w) \in \Delta$.

Note that, a function $\Pi: \Delta=\left[f_{1}, f_{2}\right] \times\left[g_{1}, g_{2}\right] \subseteq(0, \infty) \times(0, \infty) \rightarrow \mathbb{R}$ is called coordinated harmonically convex on $\Delta$ with $f_{1}<f_{2}$ and $g_{1}<g_{2}$, if the partial functions

$$
\prod_{y}:\left[f_{1}, f_{2}\right] \rightarrow \mathbb{R}, \Pi_{y}(u)=\Pi(u, y), \prod_{x}:\left[g_{1}, g_{2}\right] \rightarrow \mathbb{R}, \Pi_{x}(v)=\Pi(x, v),
$$

are harmonically convex for all $x \in\left[f_{1}, f_{2}\right]$ and $y \in\left[g_{1}, g_{2}\right]$, (for more detail, see $[9,12]$ ).

Theorem 6. [12] Let $\Pi: \Delta=\left[f_{1}, f_{2}\right] \times\left[g_{1}, g_{2}\right] \subseteq(0, \infty) \times(0, \infty) \rightarrow \mathbb{R}$ be co-ordinated harmonically convex on $\Delta$ with $f_{1}<f_{2}$ and $g_{1}<g_{2}$. Then

$$
\begin{aligned}
\prod\left(\frac{2 f_{1} f_{2}}{f_{1}+f_{2}}, \frac{2 g_{1} g_{2}}{g_{1}+g_{2}}\right) & \leq \frac{\left(f_{1} f_{2}\right)\left(g_{1} g_{2}\right)}{\left(f_{2}-f_{1}\right)\left(g_{2}-g_{1}\right)} \int_{f_{1}}^{f_{2}} \int_{g_{1}}^{g_{2}} \frac{\prod(x, y)}{x^{2} y^{2}} d y d x \\
& \leq \frac{\prod\left(f_{1}, g_{1}\right)+\prod\left(f_{1}, g_{2}\right)+\prod\left(f_{2}, g_{1}\right)+\prod\left(f_{2}, g_{2}\right)}{4} .
\end{aligned}
$$

Definition 7. [13] Let $\Pi \in L\left[f_{1}, f_{2}\right]$. The right-hand side and left-hand side Riemann- Liouville fractional integrals $J_{f_{1}+}^{\alpha} \Pi$ and $J_{f_{2}-}^{\alpha} \Pi$ of order $\alpha>0$ with $f_{2}>f_{1} \geq 0$ are defined by

$$
J_{f_{1}+}^{\alpha} \Pi(x)=\frac{1}{\Gamma(\alpha)} \int_{f_{1}}^{x}(x-t)^{\alpha-1} \prod(t) d t, x>f_{1}
$$

and

$$
J_{f_{2}-}^{\alpha} \prod(x)=\frac{1}{\Gamma(\alpha)} \int_{x}^{f_{2}}(t-x)^{\alpha-1} \prod(t) d t, x<f_{2},
$$

respectively, where $\Gamma(\alpha)$ is the Gamma function defined by $\Gamma(\alpha)=\int_{0}^{\infty} e^{-t} t^{\alpha-1} d t$.

Theorem 8. [14] Let $\prod: I \subseteq(0, \infty) \rightarrow \mathbb{R}$ be a function such that $\prod \in L_{1}\left(f_{1}, f_{2}\right)$ where $f_{1}, f_{2} \in I$ with $f_{1}<f_{2}$. If $\Pi$ is harmonocally convex function on $\left[f_{1}, f_{2}\right]$, then following inequality for fractional integral hold:

$$
\prod\left(\frac{2 f_{1} f_{2}}{f_{1}+f_{2}}\right) \leq \frac{\Gamma(\alpha+1)}{2}\left(\frac{f_{1} f_{2}}{f_{2}-f_{1}}\right)^{\alpha}\left[J_{1 / f_{1}-}^{\alpha}\left(\prod \circ \Omega\right)\left(\frac{1}{f_{2}}\right)+J_{1 / f_{2}+}^{\alpha}\left(\prod \circ \Omega\right)\left(\frac{1}{f_{1}}\right)\right] \leq \frac{\Pi\left(f_{1}\right)+\Pi\left(f_{2}\right)}{2},
$$


where $\alpha>0$ and $\Omega(x)=\frac{1}{x}$.

Definition 9. [11] Let $\Pi \in L_{1}\left(\left[f_{1}, f_{2}\right] \times\left[g_{1}, g_{2}\right]\right)$. The Riemann-Liouville integrals $J_{f_{1}+, g_{1}+^{\prime}}^{\alpha, \beta} J_{f_{1}+, g_{2}-^{\prime}}^{\alpha, \beta} J_{f_{2}-g_{1}+}^{\alpha, \beta}$ and $J_{f_{2}-, g_{2}-}^{\alpha, \beta}$ of order $\alpha, \beta>0$ with $f_{1}, g_{1} \geq 0$ are defined by

$$
\begin{aligned}
& J_{f_{1}+, g_{1}+}^{\alpha, \beta} \Pi(x, y)=\frac{1}{\Gamma(\alpha) \Gamma(\beta)} \int_{f_{1}}^{x} \int_{g_{1}}^{y}(x-t)^{\alpha-1}(y-s)^{\beta-1} \prod(t, s) d s d t, x>f_{1} y>g_{1}, \\
& J_{f_{1}+, g_{2}-}^{\alpha, \beta} \prod(x, y)=\frac{1}{\Gamma(\alpha) \Gamma(\beta)} \int_{f_{1}}^{x} \int_{y}^{g_{2}}(x-t)^{\alpha-1}(y-s)^{\beta-1} \prod(t, s) d s d t, x>f_{1} y<g_{2}, \\
& J_{f_{2}-, g_{1}+}^{\alpha, \beta} \prod(x, y)=\frac{1}{\Gamma(\alpha) \Gamma(\beta)} \int_{x}^{f_{2}} \int_{g_{1}}^{y}(x-t)^{\alpha-1}(y-s)^{\beta-1} \prod(t, s) d s d t, x<f_{2} y>g_{1},
\end{aligned}
$$

and

$$
J_{f_{2}-g_{2}-}^{\alpha, \beta} \prod(x, y)=\frac{1}{\Gamma(\alpha) \Gamma(\beta)} \int_{x}^{f_{2}} \int_{y}^{g_{2}}(x-t)^{\alpha-1}(y-s)^{\beta-1} \prod(t, s) d s d t, x<f_{2} y<g_{2},
$$

respectively. Here $\Gamma$ is the Gamma function.

Theorem 10. [11] Let $\Pi: \Delta=\left[f_{1}, f_{2}\right] \times\left[g_{1}, g_{2}\right] \subseteq \mathbb{R}^{2} \rightarrow \mathbb{R}$ be convex on $\Delta$ with $f_{1}<f_{2}$ and $g_{1}<g_{2}$ and $\Pi \in L_{1}(\Delta)$. Then

$$
\begin{aligned}
& \Pi\left(\frac{f_{1}+f_{2}}{2}, \frac{g_{1}+g_{2}}{2}\right) \leq \frac{\Gamma(\alpha+1) \Gamma(\beta+1)}{4\left(f_{2}-f_{1}\right)^{\alpha}\left(g_{2}-g_{1}\right)^{\beta}} \\
& \quad \times\left[J_{f_{1}+, g_{1}+}^{\alpha, \beta} \prod\left(f_{2}, g_{2}\right)+J_{f_{1}+, g_{2}-}^{\alpha, \beta}\left(f_{2}, g_{1}\right)+J_{f_{2}-g_{1}+}^{\alpha, \beta} \prod\left(f_{1}, g_{2}\right)+J_{f_{2}-, g_{2}-}^{\alpha, \beta} \prod\left(f_{1}, g_{1}\right)\right] \\
& \leq \frac{\prod\left(f_{1}, g_{1}\right)+\prod\left(f_{1}, g_{2}\right)+\prod\left(f_{2}, g_{1}\right)+\prod\left(f_{2}, g_{2}\right)}{4} .
\end{aligned}
$$

In this paper, we gave integral results for co-ordinated harmonically convex functions via fractional integrals.

\section{Main Results}

In this section, our aim is to prove some Hermite-Hadamard type ineqalities for co-ordinated harmonically convex functions in fractional integrals.

Theorem 11. Let $\Pi: \Delta=\left[f_{1}, f_{2}\right] \times\left[g_{1}, g_{2}\right] \subseteq(0, \infty) \times(0, \infty) \rightarrow \mathbb{R}$ be harmonically convex on $\Delta$ with $f_{1}<f_{2}$ and $g_{1}<g_{2}$ and $\Pi \in L_{1}(\Delta)$. Then

$$
\begin{aligned}
& \prod\left(\frac{2 f_{1} f_{2}}{f_{1}+f_{2}}, \frac{2 g_{1} g_{2}}{g_{1}+g_{2}}\right) \leq \frac{\Gamma(\alpha+1) \Gamma(\beta+1)}{4}\left(\frac{f_{1} f_{2}}{f_{2}-f_{1}}\right)^{\alpha}\left(\frac{g_{1} g_{2}}{g_{2}-g_{1}}\right)^{\beta} \\
& \quad \times\left[J_{1 / f_{1}-, 1 / g_{1}-}^{\alpha, \beta}\left(\prod \circ \Omega\right)\left(\frac{1}{f_{2}}, \frac{1}{g_{2}}\right)+J_{1 / f_{1}-, 1 / g_{2}+}^{\alpha, \beta}\left(\prod \circ \Omega\right)\left(\frac{1}{f_{2}}, \frac{1}{g_{1}}\right)\right. \\
& \left.\quad+J_{1 / f_{2}+, 1 / g_{1}-}^{\alpha, \beta}\left(\prod \circ \Omega\right)\left(\frac{1}{f_{1}}, \frac{1}{g_{2}}\right)+J_{1 / f_{2}+, 1 / g_{2}+}^{\alpha, \beta}\left(\prod \circ \Omega\right)\left(\frac{1}{f_{1}}, \frac{1}{g_{1}}\right)\right] \\
& \leq \frac{\prod\left(f_{1}, g_{2}\right)+\prod\left(f_{1}, g_{2}\right)+\prod\left(f_{2}, g_{1}\right)+\prod\left(f_{2}, g_{2}\right)}{4},
\end{aligned}
$$

where $\Omega(x, y)=\left(\frac{1}{x}, \frac{1}{y}\right)$ for all $(x, y) \in\left(\left[\frac{1}{f_{2}}, \frac{1}{f_{1}}\right],\left[\frac{1}{g_{2}}, \frac{1}{g_{1}}\right]\right)$.

Proof. Let $(x, y),(z, w) \in \Delta$ and $t_{1}, t_{2} \in[0,1]$. Since $\prod$ is co-ordinated harmonically convex on $\Delta$, we have 


$$
\begin{aligned}
& \prod\left(\frac{x z}{t_{1} x+\left(1-t_{1}\right) z}, \frac{y w}{t_{2} y+\left(1-t_{2}\right) w}\right) \\
& \leq t_{1} t_{2} \prod(x, y)+t_{1}\left(1-t_{2}\right) \prod(x, w)+\left(1-t_{1}\right) t_{2} \prod(z, y)+\left(1-t_{1}\right)\left(1-t_{2}\right) \prod(z, w) .
\end{aligned}
$$
get

By taking $x=\frac{f_{1} f_{2}}{t_{1} f_{1}+\left(1-t_{1}\right) f_{2}}, z=\frac{f_{1} f_{2}}{t_{1} f_{2}+\left(1-t_{1}\right) f_{1}}, y=\frac{g_{1} g_{2}}{t_{2} g_{1}+\left(1-t_{2}\right) g_{2}}, w=\frac{g_{1} g_{2}}{t_{2} g_{2}+\left(1-t_{2}\right) g_{1}}$ and $t_{1}=t_{2}=\frac{1}{2}$ in (7), we

$$
\begin{aligned}
& \prod\left(\frac{2 f_{1} f_{2}}{f_{1}+f_{2}}, \frac{2 g_{1} g_{2}}{g_{1}+g_{2}}\right) \\
& \leq \frac{1}{4}\left[\prod\left(\frac{f_{1} f_{2}}{t_{1} f_{1}+\left(1-t_{1}\right) f_{2}}, \frac{g_{1} g_{2}}{t_{2} g_{1}+\left(1-t_{2}\right) g_{2}}\right)+\prod\left(\frac{f_{1} f_{2}}{t_{1} f_{1}+\left(1-t_{1}\right) f_{2}}, \frac{g_{1} g_{2}}{t_{2} g_{2}+\left(1-t_{2}\right) g_{1}}\right)\right. \\
& \left.+\prod\left(\frac{f_{1} f_{2}}{t_{1} f_{2}+\left(1-t_{1}\right) f_{1}}, \frac{g_{1} g_{2}}{t_{2} g_{2}+\left(1-t_{2}\right) g_{1}}\right)+\prod\left(\frac{f_{1} f_{2}}{t_{1} f_{2}+\left(1-t_{1}\right) f_{1}}, \frac{g_{1} g_{2}}{t_{2} g_{1}+\left(1-t_{2}\right) g_{2}}\right)\right] .
\end{aligned}
$$

Multiplying both sides of (8) by $t_{1}^{\alpha-1} t_{2}^{\beta-1}$ and then integrating with respect to $\left(t_{1}, t_{2}\right)$ over $[0,1] \times[0,1]$, we get

$$
\begin{aligned}
\frac{1}{\alpha \beta} \prod\left(\frac{2 f_{1} f_{2}}{f_{1}+f_{2}}, \frac{2 g_{1} g_{2}}{g_{1}+g_{2}}\right) \leq & \frac{1}{4}\left[\int _ { 0 } ^ { 1 } \int _ { 0 } ^ { 1 } \left\{\prod\left(\frac{f_{1} f_{2}}{t_{1} f_{1}+\left(1-t_{1}\right) f_{2}}, \frac{g_{1} g_{2}}{t_{2} g_{1}+\left(1-t_{2}\right) g_{2}}\right)\right.\right. \\
& \left.+\prod\left(\frac{f_{1} f_{2}}{t_{1} f_{1}+\left(1-t_{1}\right) f_{2}}, \frac{g_{1} g_{2}}{t_{2} g_{2}+\left(1-t_{2}\right) g_{1}}\right)\right\} t_{1}^{\alpha-1} t_{2}^{\beta-1} d t_{1} d t_{2} \\
& +\int_{0}^{1} \int_{0}^{1}\left\{\prod\left(\frac{f_{1} f_{2}}{t_{1} f_{2}+\left(1-t_{1}\right) f_{1}}, \frac{g_{1} g_{2}}{t_{2} g_{1}+\left(1-t_{2}\right) g_{2}}\right)\right. \\
& \left.\left.+\prod\left(\frac{f_{1} f_{2}}{t_{1} f_{2}+\left(1-t_{1}\right) f_{1}}, \frac{g_{1} g_{2}}{t_{2} g_{2}+\left(1-t_{2}\right) g_{1}}\right)\right\} t_{1}^{\alpha-1} t_{2}^{\beta-1} d t_{1} d t_{2}\right] .
\end{aligned}
$$

Applying change of variable, we find

$$
\begin{aligned}
& \prod\left(\frac{2 f_{1} f_{2}}{f_{1}+f_{2}}, \frac{2 g_{1} g_{2}}{g_{1}+g_{2}}\right) \leq \frac{\alpha \beta}{4}\left(\frac{f_{1} f_{2}}{f_{2}-f_{1}}\right)^{\alpha}\left(\frac{g_{1} g_{2}}{g_{2}-g_{1}}\right)^{\beta} \\
& \times\left[\int_{1 / g_{2}}^{1 / g_{1}} \int_{1 / f_{2}}^{1 / f_{1}}\left\{\left(\frac{1}{f_{1}}-x\right)^{\alpha-1}\left(\frac{1}{g_{1}}-y\right)^{\beta-1} \prod\left(\frac{1}{x}, \frac{1}{y}\right)+\left(\frac{1}{f_{1}}-x\right)^{\alpha-1}\left(y-\frac{1}{g_{2}}\right)^{\beta-1} \prod\left(\frac{1}{x}, \frac{1}{y}\right)\right\} d x d y\right. \\
& \left.+\int_{1 / g_{2}}^{1 / g_{1}} \int_{1 / f_{2}}^{1 / f_{1}}\left\{\left(x-\frac{1}{f_{2}}\right)^{\alpha-1}\left(\frac{1}{g_{1}}-y\right)^{\beta-1} \prod\left(\frac{1}{x}, \frac{1}{y}\right)+\left(x-\frac{1}{f_{2}}\right)^{\alpha-1}\left(y-\frac{1}{g_{2}}\right)^{\beta-1} \prod\left(\frac{1}{x}, \frac{1}{y}\right)\right\} d x d y\right] .
\end{aligned}
$$

Then by multiplying and dividing by $\Gamma(\alpha) \Gamma(\beta)$ on right hand side of inequality (10), we get the first inequality of (6). For the second inequality of (6) we use the co-ordinated harmonically convexity of $\prod$ as:

$$
\begin{aligned}
& \prod\left(\frac{f_{1} f_{2}}{t_{1} f_{1}+\left(1-t_{1}\right) f_{2}}, \frac{g_{1} g_{2}}{t_{2} g_{1}+\left(1-t_{2}\right) g_{2}}\right) \\
& \leq t_{1} t_{2} \prod\left(f_{1}, g_{1}\right)+t_{1}\left(1-t_{2}\right) \prod\left(f_{1}, g_{2}\right)+\left(1-t_{1}\right) t_{2} \prod\left(f_{2}, g_{1}\right)+\left(1-t_{1}\right)\left(1-t_{2}\right) \prod\left(f_{2}, g_{2}\right), \\
& \prod\left(\frac{f_{1} f_{2}}{t_{1} f_{1}+\left(1-t_{1}\right) f_{2}}, \frac{g_{1} g_{2}}{t_{2} g_{2}+\left(1-t_{2}\right) g_{1}}\right) \\
& \leq t_{1} t_{2} \prod\left(f_{1}, g_{2}\right)+t_{1}\left(1-t_{2}\right) \prod\left(f_{1}, g_{1}\right)+\left(1-t_{1}\right) t_{2} \prod\left(f_{2}, g_{2}\right)+\left(1-t_{1}\right)\left(1-t_{2}\right) \prod\left(f_{2}, g_{1}\right),
\end{aligned}
$$




$$
\begin{aligned}
& \prod\left(\frac{f_{1} f_{2}}{t_{1} f_{2}+\left(1-t_{1}\right) f_{1}}, \frac{g_{1} g_{2}}{t_{2} g_{1}+\left(1-t_{2}\right) g_{2}}\right) \\
& \leq t_{1} t_{2} \prod\left(f_{2}, g_{1}\right)+t_{1}\left(1-t_{2}\right) \prod\left(f_{2}, g_{2}\right)+\left(1-t_{1}\right) t_{2} \prod\left(f_{1}, g_{1}\right)+\left(1-t_{1}\right)\left(1-t_{2}\right) \prod\left(f_{1}, g_{2}\right),
\end{aligned}
$$

and

$$
\begin{aligned}
& \prod\left(\frac{f_{1} f_{2}}{t_{1} f_{2}+\left(1-t_{1}\right) f_{1}}, \frac{g_{1} g_{2}}{t_{2} g_{2}+\left(1-t_{2}\right) g_{1}}\right) \\
& \leq t_{1} t_{2} \prod\left(f_{2}, g_{2}\right)+t_{1}\left(1-t_{2}\right) \prod\left(f_{2}, g_{1}\right)+\left(1-t_{1}\right) t_{2} \prod\left(f_{1}, g_{2}\right)+\left(1-t_{1}\right)\left(1-t_{2}\right) \prod\left(f_{1}, g_{1}\right) .
\end{aligned}
$$

Then by adding above inequalities, we get

$$
\begin{aligned}
& \prod\left(\frac{f_{1} f_{2}}{t_{1} f_{1}+\left(1-t_{1}\right) f_{2}}, \frac{g_{1} g_{2}}{t_{2} g_{1}+\left(1-t_{2}\right) g_{2}}\right)+\prod\left(\frac{f_{1} f_{2}}{t_{1} f_{1}+\left(1-t_{1}\right) f_{2}}, \frac{g_{1} g_{2}}{t_{2} g_{2}+\left(1-t_{2}\right) g_{1}}\right) \\
& +\prod\left(\frac{f_{1} f_{2}}{t_{1} f_{2}+\left(1-t_{1}\right) f_{1}}, \frac{g_{1} g_{2}}{t_{2} g_{1}+\left(1-t_{2}\right) g_{2}}\right)+\prod\left(\frac{f_{1} f_{2}}{t_{1} f_{2}+\left(1-t_{1}\right) f_{1}}, \frac{g_{1} g_{2}}{t_{2} g_{2}+\left(1-t_{2}\right) g_{1}}\right) \\
& \leq \prod\left(f_{1}, g_{1}\right)+\prod\left(f_{2}, g_{1}\right)+\prod\left(f_{1}, g_{2}\right)+\prod\left(f_{2}, g_{2}\right) .
\end{aligned}
$$

Thus by multiplying (11) by $t_{1}^{\alpha-1} t_{2}^{\beta-1}$ and then integrating with respect to $\left(t_{1}, t_{2}\right)$ over $[0,1] \times[0,1]$, we get the second inequality of (6). Hence the proof is completed.

Remark 1. In Theorem 11, if one takes $\alpha=\beta=1$ and using change of variable $u=1 / x$ and $v=1 / y$, then one has Theorem in [12].

Theorem 12. Let $\Pi: \Delta=\left[f_{1}, f_{2}\right] \times\left[g_{1}, g_{2}\right] \subseteq(0, \infty) \times(0, \infty) \rightarrow \mathbb{R}$ be harmonically convex on $\Delta$ with $f_{1}<f_{2}$ and $g_{1}<g_{2}$ and $\Psi \in L_{1}(\Delta)$. Then

$$
\begin{aligned}
\Pi & \left(\frac{2 f_{1} f_{2}}{f_{1}+f_{2}}, \frac{2 g_{1} g_{2}}{g_{1}+g_{2}}\right) \leq \frac{\Gamma(\alpha+1)}{4}\left(\frac{f_{1} f_{2}}{f_{2}-f_{1}}\right)^{\alpha} \\
& \times\left[J_{1 / f_{2}+}^{\alpha}\left(\prod \circ \Omega_{1}\right)\left(\frac{1}{f_{1}}, \frac{2 g_{1} g_{2}}{g_{1}+g_{2}}\right)+J_{1 / c_{1}-}^{\alpha}\left(\prod \circ \Omega_{1}\right)\left(\frac{1}{f_{2}}, \frac{2 g_{1} g_{2}}{g_{1}+g_{2}}\right)\right]+\frac{\Gamma(\beta+1)}{4}\left(\frac{g_{1} g_{2}}{g_{2}-g_{1}}\right)^{\beta} \\
& \times\left[J_{1 / g_{2}+}^{\beta}\left(\prod \circ \Omega_{2}\right)\left(\frac{2 f_{1} f_{2}}{f_{1}+f_{2}}, \frac{1}{g_{1}}\right)+J_{1 / g_{1}-}^{\beta}\left(\prod \circ \Omega_{2}\right)\left(\frac{2 f_{1} f_{2}}{f_{1}+f_{2}}, \frac{1}{g_{2}}\right)\right] \\
\leq & \frac{\Gamma(\alpha+1) \Gamma(\beta+1)}{2}\left(\frac{f_{1} f_{2}}{f_{2}-f_{1}}\right)^{\alpha}\left(\frac{g_{1} g_{2}}{g_{2}-g_{1}}\right)^{\beta} \times\left[J_{f_{1}+g_{1}+}^{\alpha, \beta}\left(\prod \circ \Omega\right)\left(\frac{1}{f_{2}}, \frac{1}{g_{1}}\right)\right. \\
& \left.+J_{f_{1}+, g_{2}-}^{\alpha, \beta}\left(\prod \circ \Omega\right)\left(\frac{1}{f_{2}}, \frac{1}{g_{1}}\right)+J_{f_{2}-g_{1}+}^{\alpha, \beta}\left(\prod \circ \Omega\right)\left(\frac{1}{f_{1}}, \frac{1}{g_{2}}\right)+J_{f_{2}-, g_{2}-}^{\alpha, \beta}\left(\prod \circ \Omega\right)\left(\frac{1}{f_{1}}, \frac{1}{g_{1}}\right)\right] \\
\leq & \frac{\Gamma(\alpha+1)}{4}\left(\frac{f_{1} f_{2}}{f_{2}-f_{1}}\right)^{\alpha}\left[J_{1 / f_{2}+}^{\alpha}\left(\prod \circ \Omega_{1}\right)\left(\frac{1}{f_{1}}, g_{2}\right)+J_{1 / f_{2}+}^{\alpha}\left(\prod \circ \Omega_{1}\right)\left(\frac{1}{f_{1}}, g_{1}\right)\right. \\
& \left.+J_{1 / f_{1}-}^{\alpha}\left(\prod \circ \Omega_{1}\right)\left(\frac{1}{f_{2}}, g_{1}\right)+J_{1 / f_{1}-}^{\alpha}\left(\prod \circ \Omega_{1}\right)\left(\frac{1}{f_{2}}, g_{1}\right)\right] \\
& +\frac{\Gamma(\beta+1)}{4}\left(\frac{g_{1} g_{2}}{g_{2}-g_{1}}\right)^{\alpha}\left[J_{1 / g_{1}-}^{\beta}\left(\prod \circ \Omega_{2}\right)\left(f_{1}, \frac{1}{g_{2}}\right)+J_{1 / g_{1}-}^{\beta}\left(\prod \circ \Omega_{2}\right)\left(f_{2}, \frac{1}{g_{2}}\right)\right. \\
& \left.+J_{1 / g_{2}+}^{\alpha}\left(\prod \circ \Omega_{2}\right)\left(f_{1}, \frac{1}{g_{1}}\right)+J_{1 / g_{2}+}^{\alpha}\left(\prod \circ \Omega_{2}\right)\left(f_{2}, \frac{1}{g_{1}}\right)\right] \\
\leq & \frac{\prod\left(f_{1}, g_{1}\right)+\prod\left(f_{1}, g_{2}\right)+\prod\left(f_{2}, g_{1}\right)+\prod\left(f_{2}, g_{2}\right)}{4},
\end{aligned}
$$

where $\Omega(x, y)=\left(\frac{1}{x}, \frac{1}{y}\right), \Omega_{1}(x, y)=\left(\frac{1}{x}, y\right)$ and $\Omega_{2}(x, y)=\left(x, \frac{1}{y}\right)$ for all $(x, y) \in\left(\left[\frac{1}{f_{2}}, \frac{1}{f_{1}}\right],\left[\frac{1}{g_{2}}, \frac{1}{g_{1}}\right]\right)$. 
Proof. Since $\Pi$ is co-ordinated harmonically convex on $\Delta$ then we have $\prod_{\frac{1}{x}}:\left[f_{1}, f_{2}\right] \rightarrow \mathbb{R}, \prod_{\frac{1}{x}}(y)=\prod\left(\frac{1}{x}, y\right)$, is harmonically convex on $\left[g_{1}, g_{2}\right]$ for all $x \in\left[\frac{1}{f_{2}}, \frac{1}{f_{1}}\right]$. Then from inequality (4), we have

$$
\begin{aligned}
& \prod_{\frac{1}{x}}\left(\frac{2 g_{1} g_{2}}{g_{1}+g_{2}}\right) \leq \frac{\Gamma(\beta+1)}{2}\left(\frac{g_{1} g_{2}}{g_{2}-g_{1}}\right)^{\beta}\left[J_{1 / c-}^{\beta}\left(\prod_{\frac{1}{x}} \circ \Omega_{2}\right)\left(\frac{1}{g_{2}}\right)+J_{1 / g_{2}+}^{\beta}\left(\prod_{\frac{1}{x}} \circ \Omega_{2}\right)\left(\frac{1}{g_{1}}\right)\right] \\
& \leq \frac{\prod_{\frac{1}{x}}\left(g_{1}\right)+\prod_{\frac{1}{x}}\left(g_{2}\right)}{2} .
\end{aligned}
$$

In other words,

$$
\begin{gathered}
\prod\left(\frac{1}{x}, \frac{2 g_{1} g_{2}}{g_{1}+g_{2}}\right) \leq \frac{\beta}{2}\left(\frac{g_{1} g_{2}}{g_{2}-g_{1}}\right)^{\beta}\left[\int_{1 / g_{2}}^{1 / g_{1}}\left(y-\frac{1}{g_{2}}\right)^{\beta-1} \prod\left(\frac{1}{x}, \frac{1}{y}\right) \mathrm{d} y\right. \\
\left.+\int_{1 / g_{2}}^{1 / g_{1}}\left(\frac{1}{g_{1}}-y\right)^{\beta-1} \prod\left(\frac{1}{x}, \frac{1}{y}\right) \mathrm{d} y\right] \leq \frac{\Pi\left(\frac{1}{x}, g_{1}\right)+\Pi\left(\frac{1}{x}, g_{2}\right)}{2},
\end{gathered}
$$

for all $x \in\left[\frac{1}{f_{2}}, \frac{1}{f_{1}}\right]$. Now by multiplying (14) by $\frac{\alpha\left(x-1 / f_{2}\right)^{\alpha-1}}{2}\left(\frac{f_{1} f_{2}}{f_{2}-f_{1}}\right)^{\alpha}$ and $\frac{\alpha\left(1 / f_{1}-x\right)^{\alpha-1}}{2}\left(\frac{f_{1} f_{2}}{f_{2}-f_{1}}\right)^{\alpha}$, and then integrating with respect to $x$ over $\left[1 / f_{2}, 1 / f_{1}\right]$, respectively, we find

$$
\begin{aligned}
& \frac{\alpha}{2}\left(\frac{f_{1} f_{2}}{f_{2}-f_{1}}\right)^{\alpha} \int_{1 / f_{2}}^{1 / f_{1}}\left(x-\frac{1}{f_{2}}\right)^{\alpha-1} \prod\left(\frac{1}{x}, \frac{2 g_{1} g_{2}}{g_{1}+g_{2}}\right) \mathrm{d} x \leq \frac{\alpha \beta}{4}\left(\frac{f_{1} f_{2}}{f_{2}-f_{1}}\right)^{\alpha}\left(\frac{g_{1} g_{2}}{g_{2}-g_{1}}\right)^{\beta} \\
& \quad \times\left[\int_{1 / f_{2}}^{1 / f_{1}} \int_{1 / g_{2}}^{1 / g_{1}}\left(x-\frac{1}{f_{2}}\right)^{\alpha-1}\left(y-\frac{1}{g_{2}}\right)^{\beta-1} \prod\left(\frac{1}{x}, \frac{1}{y}\right) \mathrm{d} y \mathrm{~d} x\right. \\
& \left.\quad+\int_{1 / f_{2}}^{1 / f_{1}} \int_{1 / g_{2}}^{1 / g_{1}}\left(x-\frac{1}{f_{2}}\right)^{\alpha-1}\left(\frac{1}{g_{1}}-y\right)^{\beta-1} \prod\left(\frac{1}{x}, \frac{1}{y}\right) \mathrm{d} y \mathrm{~d} x\right] \\
& \leq \frac{\alpha \beta}{4}\left(\frac{f_{1} f_{2}}{f_{2}-f_{1}}\right)^{\alpha}\left[\int_{1 / f_{2}}^{1 / f_{1}}\left(x-\frac{1}{f_{2}}\right)^{\alpha-1} \prod\left(\frac{1}{x}, g_{1}\right) \mathrm{d} x+\int_{1 / f_{2}}^{1 / f_{1}}\left(x-\frac{1}{f_{2}}\right)^{\alpha-1} \prod\left(\frac{1}{x}, g_{2}\right) \mathrm{d} x\right],
\end{aligned}
$$

and

$$
\begin{aligned}
& \frac{\alpha}{2}\left(\frac{f_{1} f_{2}}{f_{2}-f_{1}}\right)^{\alpha} \int_{1 / f_{2}}^{1 / f_{1}}\left(\frac{1}{f_{1}}-x\right)^{\alpha-1} \prod\left(\frac{1}{x}, \frac{2 g_{1} g_{2}}{g_{1}+g_{2}}\right) \mathrm{d} x \leq \frac{\alpha \beta}{4}\left(\frac{f_{1} f_{2}}{f_{2}-f_{1}}\right)^{\alpha}\left(\frac{g_{1} g_{2}}{g_{2}-g_{1}}\right)^{\beta} \\
& \quad \times\left[\int_{1 / f_{2}}^{1 / f_{1}} \int_{1 / g_{2}}^{1 / g_{1}}\left(\frac{1}{f_{1}}-x\right)^{\alpha-1}\left(y-\frac{1}{g_{2}}\right)^{\beta-1} \prod\left(\frac{1}{x}, \frac{1}{y}\right) \mathrm{d} y \mathrm{~d} x\right. \\
& \left.\quad+\int_{1 / f_{2}}^{1 / f_{1}} \int_{1 / g_{2}}^{1 / g_{1}}\left(\frac{1}{f_{1}}-x\right)^{\alpha-1}\left(\frac{1}{g_{1}}-y\right)^{\beta-1} \prod\left(\frac{1}{x}, \frac{1}{y}\right) \mathrm{d} y \mathrm{~d} x\right] \\
& \leq \frac{\alpha \beta}{4}\left(\frac{f_{1} f_{2}}{f_{2}-f_{1}}\right)^{\alpha}\left[\int_{1 / f_{2}}^{1 / f_{1}}\left(\frac{1}{f_{1}}-x\right)^{\alpha-1} \prod\left(\frac{1}{x}, g_{1}\right) \mathrm{d} x+\int_{1 / f_{2}}^{1 / f_{1}}\left(\frac{1}{f_{1}}-x\right)^{\alpha-1} \prod\left(\frac{1}{x}, g_{2}\right) \mathrm{d} x\right] .
\end{aligned}
$$

Again by similar arguments for $\prod_{\frac{1}{y}}:\left[f_{1}, f_{2}\right] \rightarrow \mathbb{R}, \prod_{\frac{1}{y}}(x)=\prod\left(x, \frac{1}{y}\right)$, we get

$$
\begin{aligned}
& \frac{\beta}{2}\left(\frac{g_{1} g_{2}}{g_{2}-g_{1}}\right)^{\beta} \int_{1 / g_{2}}^{1 / g_{1}}\left(y-\frac{1}{g_{2}}\right)^{\beta-1} \prod\left(\frac{2 f_{1} f_{2}}{f_{1}+f_{2}}, \frac{1}{y}\right) \mathrm{d} y \\
& \leq \frac{\alpha \beta}{4}\left(\frac{f_{1} f_{2}}{f_{2}-f_{1}}\right)^{\alpha}\left(\frac{g_{1} g_{2}}{g_{2}-g_{1}}\right)^{\beta}\left[\int_{1 / f_{2}}^{1 / f_{1}} \int_{1 / g_{2}}^{1 / g_{1}}\left(u-\frac{1}{f_{2}}\right)^{\alpha-1}\left(y-\frac{1}{g_{2}}\right)^{\beta-1} \prod\left(\frac{1}{x}, \frac{1}{y}\right) \mathrm{d} y \mathrm{~d} x\right. \\
& \left.\quad+\int_{1 / f_{2}}^{1 / f_{1}} \int_{1 / g_{2}}^{1 / g_{1}}\left(\frac{1}{f_{1}}-x\right)^{\alpha-1}\left(y-\frac{1}{g_{2}}\right)^{\beta-1} \prod\left(\frac{1}{x}, \frac{1}{y}\right) \mathrm{d} y \mathrm{~d} x\right]
\end{aligned}
$$




$$
\leq \frac{\alpha \beta}{4}\left(\frac{f_{1} f_{2}}{f_{2}-f_{1}}\right)^{\alpha}\left[\int_{1 / g_{2}}^{1 / g_{1}}\left(y-\frac{1}{g_{2}}\right)^{\alpha-1} \prod\left(f_{1}, \frac{1}{y}\right) \mathrm{d} y+\int_{1 / g_{2}}^{1 / g_{1}}\left(y-\frac{1}{g_{2}}\right)^{\beta-1} \prod\left(f_{2}, \frac{1}{y}\right) \mathrm{d} y\right]
$$

and

$$
\begin{aligned}
& \frac{\beta}{2}\left(\frac{g_{1} g_{2}}{g_{2}-g_{1}}\right)^{\beta} \int_{1 / g_{2}}^{1 / g_{1}}\left(\frac{1}{g_{1}}-y\right)^{\beta-1} \prod\left(\frac{2 f_{1} f_{2}}{f_{1}+f_{2}}, \frac{1}{y}\right) \mathrm{d} y \\
& \leq \frac{\alpha \beta}{4}\left(\frac{f_{1} f_{2}}{f_{2}-f_{1}}\right)^{\alpha}\left(\frac{g_{1} g_{2}}{g_{2}-g_{1}}\right)^{\beta}\left[\int_{1 / f_{2}}^{1 / f_{1}} \int_{1 / g_{2}}^{1 / g_{1}}\left(x-\frac{1}{f_{2}}\right)^{\alpha-1}\left(\frac{1}{g_{1}}-y\right)^{\beta-1} \prod\left(\frac{1}{x}, \frac{1}{y}\right) \mathrm{d} y \mathrm{~d} x\right. \\
&\left.\quad+\int_{1 / f_{2}}^{1 / f_{1}} \int_{1 / g_{2}}^{1 / g_{1}}\left(\frac{1}{f_{1}}-x\right)^{\alpha-1}\left(\frac{1}{g_{1}}-y\right)^{\beta-1} \prod\left(\frac{1}{x}, \frac{1}{y}\right) \mathrm{d} y \mathrm{~d} x\right] \\
& \leq \frac{\alpha \beta}{4}\left(\frac{f_{1} f_{2}}{f_{2}-f_{1}}\right)^{\alpha}\left[\int_{1 / g_{2}}^{1 / g_{1}}\left(\frac{1}{g_{1}}-y\right)^{\alpha-1} \prod\left(f_{1}, \frac{1}{y}\right) \mathrm{d} y+\int_{1 / g_{2}}^{1 / g_{1}}\left(\frac{1}{g_{1}}-y\right)^{\beta-1} \prod\left(f_{2}, \frac{1}{y}\right) \mathrm{d} y\right] .
\end{aligned}
$$

By adding inequalities (15)-(18), we have

$$
\begin{aligned}
& \frac{\Gamma(\alpha+1)}{4}\left(\frac{f_{1} f_{2}}{f_{2}-f_{1}}\right)^{\alpha}\left[J_{1 / f_{2}+}^{\alpha}\left(\prod \circ \Omega_{1}\right)\left(\frac{1}{f_{1}}, \frac{2 g_{1} g_{2}}{g_{1}+g_{2}}\right)+J_{1 / c_{1}-}^{\alpha}\left(\prod \circ \Omega_{1}\right)\left(\frac{1}{f_{2}}, \frac{2 g_{1} g_{2}}{g_{1}+g_{2}}\right)\right] \\
& \quad+\frac{\Gamma(\beta+1)}{4}\left(\frac{g_{1} g_{2}}{g_{2}-g_{1}}\right)^{\beta}\left[J_{1 / g_{2}+}^{\beta}\left(\prod \circ \Omega_{2}\right)\left(\frac{2 f_{1} f_{2}}{f_{1}+f_{2}}, \frac{1}{g_{1}}\right)+J_{1 / g_{1}-}^{\beta}\left(\prod \circ \Omega_{2}\right)\left(\frac{2 f_{1} f_{2}}{f_{1}+f_{2}}, \frac{1}{g_{2}}\right)\right] \\
& \leq \frac{\Gamma(\alpha+1) \Gamma(\beta+1)}{2}\left(\frac{f_{1} f_{2}}{f_{2}-f_{1}}\right)^{\alpha}\left(\frac{g_{1} g_{2}}{g_{2}-g_{1}}\right)^{\beta} \times\left[J_{f_{1}+g_{1}+}^{\alpha, \beta}\left(\prod \circ \Omega\right)\left(\frac{1}{f_{2}}, \frac{1}{g_{1}}\right)+J_{f_{1}+, g_{2}-}^{\alpha, \beta}\left(\prod \circ \Omega\right)\left(\frac{1}{f_{2}}, \frac{1}{g_{1}}\right)\right. \\
& \left.\quad+J_{f_{2}-g_{1}+}^{\alpha, \beta}\left(\prod \circ \Omega\right)\left(\frac{1}{f_{1}}, \frac{1}{g_{2}}\right)+J_{f_{2}-g_{2}-}^{\alpha, \beta}\left(\prod \circ \Omega\right)\left(\frac{1}{f_{1}}, \frac{1}{g_{1}}\right)\right] \\
& \leq \frac{\Gamma(\alpha+1)}{4}\left(\frac{f_{1} f_{2}}{f_{2}-f_{1}}\right)^{\alpha}\left[J_{1 / f_{2}+}^{\alpha}\left(\prod \circ \Omega_{1}\right)\left(\frac{1}{f_{1}}, g_{2}\right)+J_{1 / f_{2}+}^{\alpha}\left(\prod \circ \Omega_{1}\right)\left(\frac{1}{f_{1}}, g_{1}\right)\right. \\
& \left.\quad+J_{1 / f_{1}-}^{\alpha}\left(\prod \circ \Omega_{1}\right)\left(\frac{1}{f_{2}}, g_{1}\right)+J_{1 / f_{1}-}^{\alpha}\left(\prod \circ \Omega_{1}\right)\left(\frac{1}{f_{2}}, g_{1}\right)\right] \\
& \quad+\frac{\Gamma(\beta+1)}{4}\left(\frac{g_{1} g_{2}}{g_{2}-g_{1}}\right)^{\alpha}\left[J_{1 / g_{1}-}^{\beta}\left(\prod \circ \Omega_{2}\right)\left(f_{1}, \frac{1}{g_{2}}\right)+J_{1 / g_{1}-}^{\beta}\left(\prod \circ \Omega_{2}\right)\left(f_{2}, \frac{1}{g_{2}}\right)\right. \\
& \left.\quad+J_{1 / g_{2}+}^{\alpha}\left(\prod \circ \Omega_{2}\right)\left(f_{1}, \frac{1}{g_{1}}\right)+J_{1 / g_{2}+}^{\alpha}\left(\prod \circ \Omega_{2}\right)\left(f_{2}, \frac{1}{g_{1}}\right)\right] .
\end{aligned}
$$

This completes the second and third inequality of (12). Now again using (4), we have

$$
\begin{aligned}
& \prod\left(\frac{2 f_{1} f_{2}}{f_{1}+f_{2}}, \frac{2 g_{1} g_{2}}{g_{1}+g_{2}}\right) \leq \frac{\alpha}{2}\left(\frac{f_{1} f_{2}}{f_{2}-f_{1}}\right)^{\alpha}\left[\int_{1 / f_{2}}^{1 / f-1}\left(\frac{1}{f_{1}}-x\right)^{\alpha-1} \prod\left(\frac{1}{x}, \frac{2 g_{1} g_{2}}{g_{1}+g_{2}}\right) \mathrm{d} x\right. \\
& \left.\quad+\int_{1 / f_{2}}^{1 / f_{1}}\left(x-\frac{1}{f_{2}}\right)^{\beta-1} \prod\left(\frac{1}{x}, \frac{2 g_{1} g_{2}}{g_{1}+g_{2}}\right) \mathrm{d} x\right] \\
& \prod\left(\frac{2 f_{1} f_{2}}{f_{1}+f_{2}}, \frac{2 g_{1} g_{2}}{g_{1}+g_{2}}\right) \leq \frac{\beta}{2}\left(\frac{g_{1} g_{2}}{g_{2}-g_{1}}\right)^{\beta}\left[\int_{1 / g_{2}}^{1 / g_{1}}\left(\frac{1}{g_{1}}-y\right)^{\beta-1} \prod\left(\frac{2 f_{1} f_{2}}{f_{1}+f_{2}}, \frac{1}{y}\right) \mathrm{d} y\right. \\
& \left.\quad+\int_{1 / g_{2}}^{1 / g_{1}}\left(y-\frac{1}{g_{2}}\right)^{\beta-1} \prod\left(\frac{2 f_{1} f_{2}}{f_{1}+f_{2}}, \frac{1}{y}\right) \mathrm{d} y\right] .
\end{aligned}
$$


Adding (20) and (21), we get

$$
\begin{aligned}
& \Pi\left(\frac{2 f_{1} f_{2}}{f_{1}+f_{2}}, \frac{2 g_{1} g_{2}}{g_{1}+g_{2}}\right) \\
& \leq \frac{\Gamma(\alpha+1)}{4}\left(\frac{f_{1} f_{2}}{f_{2}-f_{1}}\right)^{\alpha}\left[J_{1 / f_{2}+}^{\alpha}\left(\prod \circ \Omega_{1}\right)\left(\frac{1}{f_{1}}, \frac{2 g_{1} g_{2}}{g_{1}+g_{2}}\right)+J_{1 / f_{1}-}^{\alpha}\left(\prod \circ \Omega_{1}\right)\left(\frac{1}{f_{2}}, \frac{2 g_{1} g_{2}}{g_{1}+g_{2}}\right)\right] \\
& \quad+\frac{\Gamma(\beta+1)}{4}\left(\frac{g_{1} g_{2}}{g_{2}-g_{1}}\right)^{\beta} \times\left[J_{1 / g_{2}+}^{\beta}\left(\prod \circ \Omega_{2}\right)\left(\frac{2 f_{1} f_{2}}{f_{1}+f_{2}}, \frac{1}{g_{1}}\right)+J_{1 / g_{1}-}^{\beta}\left(\prod \circ \Omega_{2}\right)\left(\frac{2 f_{1} f_{2}}{f_{1}+f_{2}}, \frac{1}{g_{2}}\right)\right] .
\end{aligned}
$$

This completes the first inequality of (12). For the last inequality by using (4), we have

$$
\begin{aligned}
& \frac{\alpha}{2}\left(\frac{f_{1} f_{2}}{f_{2}-f_{1}}\right)^{\alpha}\left[\int_{1 / f_{2}}^{1 / f_{1}}\left(\frac{1}{f_{1}}-x\right)^{\alpha-1} \Pi\left(\frac{1}{x}, g_{1}\right) \mathrm{d} x+\int_{1 / f_{2}}^{1 / f_{1}}\left(x-\frac{1}{f_{2}}\right)^{\beta-1} \Pi\left(\frac{1}{x}, g_{1}\right) \mathrm{d} x\right] \\
& \leq \frac{\prod\left(f_{1}, g_{1}\right)+\prod\left(f_{2}, g_{1}\right)}{2}, \\
& \frac{\alpha}{2}\left(\frac{f_{1} f_{2}}{f_{2}-f_{1}}\right)^{\alpha}\left[\int_{1 / f_{2}}^{1 / f_{1}}\left(\frac{1}{f_{1}}-x\right)^{\alpha-1} \Pi\left(\frac{1}{x}, g_{2}\right) \mathrm{d} x+\int_{1 / f_{2}}^{1 / f_{1}}\left(x-\frac{1}{f_{2}}\right)^{\beta-1} \Pi\left(\frac{1}{x}, g_{2}\right) \mathrm{d} x\right] \\
& \leq \frac{\prod\left(f_{1}, g_{2}\right)+\prod\left(f_{2}, g_{2}\right)}{2}, \\
& \frac{\beta}{2}\left(\frac{g_{1} g_{2}}{g_{2}-g_{1}}\right)^{\beta}\left[\int_{1 / g_{2}}^{1 / g_{1}}\left(\frac{1}{g_{1}}-y\right)^{\beta-1} \prod\left(f_{1}, \frac{1}{y}\right) \mathrm{d} y+\int_{1 / g_{2}}^{1 / g_{1}}\left(y-\frac{1}{g_{2}}\right)^{\beta-1} \prod\left(f_{1}, \frac{1}{y}\right) \mathrm{d} y\right] \\
& \leq \frac{\prod\left(f_{1}, g_{1}\right)+\prod\left(f_{1}, g_{2}\right)}{2}, \\
& \frac{\beta}{2}\left(\frac{g_{1} g_{2}}{g_{2}-g_{1}}\right)^{\beta}\left[\int_{1 / g_{2}}^{1 / g_{1}}\left(\frac{1}{g_{1}}-y\right)^{\beta-1} \prod\left(f_{2}, \frac{1}{y}\right) \mathrm{d} y+\int_{1 / g_{2}}^{1 / g_{1}}\left(y-\frac{1}{g_{2}}\right)^{\beta-1} \prod\left(f_{2}, \frac{1}{y}\right) \mathrm{d} y\right] \\
& \leq \frac{\prod\left(f_{2}, g_{1}\right)+\prod\left(f_{2}, g_{2}\right)}{2} .
\end{aligned}
$$

Thus by adding all above inequalities, we get the last inequality of (12). Hence the proof is completed.

Lemma 1. Let $\Pi: \Delta=\left[f_{1}, f_{2}\right] \times\left[g_{1}, g_{2}\right] \subseteq(0, \infty) \times(0, \infty) \rightarrow \mathbb{R}$ be a partial differentiable mapping on $\Delta$ with $0<f_{1}<f_{2}$ and $0<g_{1}<g_{2}$. If $\partial^{2} \Pi / \partial t_{1} \partial t_{2} \in L_{1}(\Delta)$, then following holds:

$$
\begin{aligned}
& \frac{\prod\left(f_{1}, g_{1}\right)+\Pi\left(f_{1}, g_{2}\right)+\Pi\left(f_{2}, g_{1}\right)+\Pi\left(f_{2}, g_{2}\right)}{4}+\frac{\Gamma(\alpha+1) \Gamma(\beta+1)}{4}\left(\frac{f_{1} f_{2}}{f_{2}-f_{1}}\right)^{\alpha}\left(\frac{g_{1} g_{2}}{g_{2}-g_{1}}\right)^{\beta} \\
& \quad \times\left[J_{1 / f_{2}+, 1 / g_{1}+}^{\alpha, \beta}\left(\prod \circ \Omega\right)\left(\frac{1}{f_{1}}, \frac{1}{g_{1}}\right)+J_{1 / f_{1}-, 1 / g_{2}+}^{\alpha, \beta}\left(\prod \circ \Omega\right)\left(\frac{1}{f_{2}}, \frac{1}{g_{1}}\right)\right. \\
& \left.\quad+J_{1 / f_{2}+, 1 / g_{1}-}^{\alpha, \beta}\left(\prod \circ \Omega\right)\left(\frac{1}{f_{1}}, \frac{1}{g_{2}}\right)+J_{1 / f_{1}-, 1 / g_{1}-}^{\alpha, \beta}\left(\prod \circ \Omega\right)\left(\frac{1}{f_{2}}, \frac{1}{g_{2}}\right)\right]-\Xi \\
& =\frac{f_{1} f_{2} g_{1} g_{2}\left(f_{2}-f_{1}\right)\left(g_{2}-g_{1}\right)}{4}\left[\int_{0}^{1} \int_{0}^{1} \frac{r_{1}^{\alpha} r_{2}^{\beta}}{A_{t_{1}}^{2} B_{t_{2}}^{2}} \frac{\partial^{2} \Pi}{\partial t_{1} \partial t_{2}}\left(\frac{f_{1} f_{2}}{A_{t_{1}}}, \frac{g_{1} g_{2}}{B_{t_{2}}}\right) d t_{2} d t_{1}\right. \\
& \quad-\int_{0}^{1} \int_{0}^{1} \frac{\left(1-t_{1}\right)^{\alpha} t_{2}^{\beta}}{A_{t_{1}}^{2} B_{t_{2}}^{2}} \frac{\partial^{2} \Pi}{\partial t_{1} \partial t_{2}}\left(\frac{f_{1} f_{2}}{A_{t_{1}}}, \frac{g_{1} g_{2}}{B_{t_{2}}}\right) d t_{2} d t_{1}-\int_{0}^{1} \int_{0}^{1} \frac{t_{1}^{\alpha}\left(1-t_{2}\right)^{\beta}}{A_{t_{1}}^{2} B_{t_{2}}^{2}} \frac{\partial^{2} \Pi}{\partial t_{1} \partial t_{2}}\left(\frac{f_{1} f_{2}}{A_{t_{1}}}, \frac{g_{1} g_{2}}{B_{t_{2}}}\right) d t_{2} d t_{1} \\
& \left.+\int_{0}^{1} \int_{0}^{1} \frac{\left(1-t_{1}\right)^{\alpha}\left(1-t_{2}\right)^{\beta}}{A_{t_{1}}^{2} B_{t_{2}}^{2}} \frac{\partial^{2} \Pi}{\partial t_{1} \partial t_{2}}\left(\frac{f_{1} f_{2}}{A_{t_{1}}}, \frac{g_{1} g_{2}}{B_{t_{2}}}\right) d t_{2} d t_{1}\right],
\end{aligned}
$$

where 


$$
\begin{aligned}
\Xi= & \frac{\Gamma(\alpha+1)}{4}\left(\frac{f_{1} f_{2}}{f_{2}-f_{1}}\right)^{\alpha}\left[J_{1 / f_{2}+}^{\alpha}\left(\prod \circ \Omega_{1}\right)\left(\frac{1}{f_{1}}, g_{2}\right)+J_{1 / f_{1}-}^{\alpha}\left(\prod \circ \Omega_{1}\right)\left(\frac{1}{f_{2}}, g_{2}\right)+J_{1 / f_{2}+}^{\alpha}\left(\prod \circ \Omega_{1}\right)\left(\frac{1}{f_{1}}, g_{1}\right)\right. \\
& \left.+J_{1 / f_{1}-}^{\alpha}\left(\prod \circ \Omega_{1}\right)\left(\frac{1}{f_{2}}, g_{1}\right)\right]+\frac{\Gamma(\beta+1)}{4}\left(\frac{g_{1} g_{2}}{g_{2}-g_{1}}\right)^{\beta}\left[J_{1 / g_{2}+}^{\beta}\left(\prod \circ \Omega_{2}\right)\left(f_{2}, \frac{1}{g_{1}}\right)\right. \\
& \left.+J_{1 / d_{2}+}^{\beta}\left(\prod \circ \Omega_{2}\right)\left(f_{1}, \frac{1}{g_{1}}\right)+J_{1 / d_{1}-}^{\beta}\left(\prod \circ \Omega_{2}\right)\left(f_{2}, \frac{1}{g_{2}}\right)+J_{1 / g_{1}-}^{\beta}\left(\prod \circ \Omega_{2}\right)\left(f_{1}, \frac{1}{g_{2}}\right)\right],
\end{aligned}
$$

and $A_{t_{1}}=t_{1} f_{1}+\left(1-t_{1}\right) f_{2}, B_{t_{2}}=t_{2} c+\left(1-t_{2}\right) d$. Also, $g(x, y)=\left(\frac{1}{x}, \frac{1}{y}\right), g_{1}(x, y)=\left(\frac{1}{x}, y\right)$, and $g_{2}(x, y)=\left(x, \frac{1}{y}\right)$ for all $(x, y) \in \Delta$.

Proof. By integration by parts and using the change of variable $x=\frac{A_{t_{1}}}{f_{1} f_{2}}$ and $y=\frac{B_{t_{2}}}{g_{1} g_{2}}$, we find that

$$
\begin{aligned}
I_{1}= & \int_{0}^{1} \int_{0}^{1} \frac{t_{1}^{\alpha} t_{2}^{\beta}}{A_{t_{1}}^{2} B_{t_{2}}^{2}} \frac{\partial^{2} \Pi}{\partial t_{1} \partial t_{2}}\left(\frac{f_{1} f_{2}}{A_{t_{1}}}, \frac{g_{1} g_{2}}{B_{t_{2}}}\right) \mathrm{d} t_{2} \mathrm{~d} t_{1} \\
= & \int_{0}^{1} \frac{t_{2}^{\beta}}{B_{t_{2}}^{2}}\left\{\left.\frac{t_{1}^{\alpha}}{f_{1} f_{2}\left(f_{2}-f_{1}\right)} \frac{\partial \prod}{\partial t_{2}}\left(\frac{f_{1} f_{2}}{A_{t_{1}}}, \frac{g_{1} g_{2}}{B_{t_{2}}}\right)\right|_{0} ^{1}-\frac{\alpha}{f_{1} f_{2}\left(f_{2}-f_{1}\right)} \int_{0}^{1} t_{1}^{\alpha-1} \frac{\partial \prod}{\partial t_{2}}\left(\frac{f_{1} f_{2}}{A_{t_{1}}}, \frac{g_{1} g_{2}}{B_{t_{2}}}\right) \mathrm{d} t_{1}\right\} \mathrm{d} t_{2} \\
= & \frac{1}{f_{1} f_{2}\left(f_{2}-f_{1}\right)} \int_{0}^{1} \frac{t_{2}^{\beta}}{B_{t_{2}}^{2}} \frac{\partial \prod}{\partial t_{2}}\left(f_{2}, \frac{g_{1} g_{2}}{B_{t_{2}}}\right) \mathrm{d} t_{2}-\frac{\alpha}{f_{1} f_{2}\left(f_{2}-f_{1}\right)} \int_{0}^{1} t_{1}^{\alpha-1}\left\{\int_{0}^{1} \frac{t_{2}^{\beta}}{B_{t_{2}}^{2}} \frac{\partial \prod}{\partial t_{2}}\left(\frac{f_{1} f_{2}}{A_{t_{1}}}, \frac{g_{1} g_{2}}{B_{t_{2}}}\right) \mathrm{d} t_{2}\right\} \mathrm{d} t_{1} \\
= & \frac{1}{f_{1} f_{2} g_{1} g_{2}\left(f_{2}-f_{1}\right)\left(g_{2}-g_{1}\right)} \prod\left(f_{2}, g_{2}\right)-\frac{\beta}{f_{1} f_{2} g_{1} g_{2}\left(f_{2}-f_{1}\right)\left(g_{2}-g_{1}\right)} \int_{0}^{1} t_{2}^{\beta-1} \prod\left(f_{2}, \frac{g_{1} g_{2}}{B_{t_{2}}}\right) \mathrm{d} t_{2} \\
& -\frac{\alpha}{f_{1} f_{2} g_{1} g_{2}\left(f_{2}-f_{1}\right)\left(g_{2}-g_{1}\right)} \int_{0}^{1} t_{1}^{\alpha-1} \prod\left(\frac{f_{1} f_{2}}{A_{t_{1}}}, d\right) \mathrm{d} t_{1} \\
& +\frac{\alpha \beta}{f_{1} f_{2} g_{1} g_{2}\left(f_{2}-f_{1}\right)\left(g_{2}-g_{1}\right)} \int_{0}^{1} t_{1}^{\alpha-1} t_{2}^{\beta-1} \prod\left(\frac{f_{1} f_{2}}{A_{r_{1}}}, \frac{g_{1} g_{2}}{B_{t_{2}}}\right) \mathrm{d} t_{2} \\
= & \frac{1}{f_{1} f_{2} g_{1} g_{2}\left(f_{2}-f_{1}\right)\left(g_{2}-g_{1}\right)} \times\left[\prod\left(f_{2}, g_{2}\right)-\Gamma(\beta+1)\left(\frac{g_{1} g_{2}}{g_{2}-g_{1}}\right)^{\beta} J_{1 / g_{2}+}^{\beta}\left(\prod \circ \Omega_{2}\right)\left(f_{2}, \frac{1}{g_{1}}\right)\right. \\
& -\Gamma(\alpha+1)\left(\frac{f_{1} f_{2}}{f_{2}-f_{1}}\right)^{\alpha} J_{1 / f_{2}+}^{\alpha}\left(\prod \circ \Omega_{1}\right)\left(\frac{1}{f_{1}}, g_{2}\right)+\Gamma(\alpha+1) \Gamma(\beta+1) \\
& \left.\times\left(\frac{f_{1} f_{2}}{f_{2}-f_{1}}\right)^{\alpha}\left(\frac{g_{1} g_{2}}{g_{2}-g_{1}}\right)^{\beta} J_{1 / f_{2}+, 1 / g_{2}+}^{\alpha, \beta}\left(\prod \circ \Omega\right)\left(\frac{1}{f_{1}}, \frac{1}{g_{1}}\right)\right] .
\end{aligned}
$$

Similarly, we can have

$$
\begin{aligned}
& I_{2}= \int_{0}^{1} \int_{0}^{1} \frac{\left(1-t_{1}\right)^{\alpha} t_{2}^{\beta}}{A_{t_{1}}^{2} B_{t_{2}}^{2}} \frac{\partial^{2} \Pi}{\partial t_{1} \partial t_{2}}\left(\frac{f_{1} f_{2}}{A_{t_{1}}}, \frac{g_{1} g_{2}}{B_{t_{2}}}\right) \mathrm{d} t_{2} \mathrm{~d} t_{1} \\
&= \frac{1}{f_{1} f_{2} g_{1} g_{2}\left(f_{2}-f_{1}\right)\left(g_{2}-g_{1}\right)}\left[-\prod\left(f_{1}, g_{1}\right)+\Gamma(\beta+1)\left(\frac{g_{1} g_{2}}{g_{2}-g_{1}}\right)^{\beta} J_{1 / g_{2}+}^{\beta}\left(\prod \circ \Omega_{2}\right)\left(f_{1}, \frac{1}{g_{1}}\right)\right. \\
&+\Gamma(\alpha+1)\left(\frac{f_{1} f_{2}}{f_{2}-f_{1}}\right)^{\alpha} J_{1 / f_{1}-}^{\alpha}\left(\prod \circ \Omega_{1}\right)\left(\frac{1}{f_{2}}, g_{2}\right)-\Gamma(\alpha+1) \Gamma(\beta+1) \\
&\left.\times\left(\frac{f_{1} f_{2}}{f_{2}-f_{1}}\right)^{\alpha}\left(\frac{g_{1} g_{2}}{g_{2}-g_{1}}\right)^{\beta} J_{1 / f_{2}+, 1 / g_{2}+}^{\alpha, \beta}\left(\prod \circ \Omega\right)\left(\frac{1}{f_{1}}, \frac{1}{g_{1}}\right)\right] . \\
& I_{3}=\int_{0}^{1} \int_{0}^{1} \frac{t_{1}^{\alpha}\left(1-t_{2}\right)^{\beta}}{A_{t_{1}}^{2} B_{t_{2}}^{2}} \frac{\partial^{2} \Pi}{\partial t_{1} \partial t_{2}}\left(\frac{f_{1} f_{2}}{A_{t_{1}}}, \frac{g_{1} g_{2}}{B_{t_{2}}}\right) \mathrm{d} t_{2} \mathrm{~d} t_{1}
\end{aligned}
$$




$$
\begin{aligned}
= & \frac{1}{f_{1} f_{2} g_{1} g_{2}\left(f_{2}-f_{1}\right)\left(g_{2}-g_{1}\right)}\left[-\prod\left(f_{2}, g_{1}\right)+\Gamma(\beta+1)\left(\frac{g_{1} g_{2}}{g_{2}-g_{1}}\right)^{\beta} J_{1 / g_{1}-}^{\beta}\left(\prod \circ \Omega_{2}\right)\left(f_{2}, \frac{1}{g_{2}}\right)\right. \\
& +\Gamma(\alpha+1)\left(\frac{f_{1} f_{2}}{f_{2}-f_{1}}\right)^{\alpha} J_{1 / f_{2}+}^{\alpha}\left(\prod \circ \Omega_{1}\right)\left(\frac{1}{f_{1}}, g_{1}\right)-\Gamma(\alpha+1) \Gamma(\beta+1) \\
& \left.\times\left(\frac{f_{1} f_{2}}{f_{2}-f_{1}}\right)^{\alpha}\left(\frac{g_{1} g_{2}}{g_{2}-g_{1}}\right)^{\beta} J_{1 / f_{2}+, 1 / g_{1}-}^{\alpha, \beta}\left(\prod \circ \Omega\right)\left(\frac{1}{f_{1}}, \frac{1}{g_{2}}\right)\right] . \\
I_{4}= & \int_{0}^{1} \int_{0}^{1} \frac{\left(1-t_{1}\right)^{\alpha}\left(1-t_{2}\right)^{\beta}}{A_{t_{1}}^{2} B_{t_{2}}^{2}} \frac{\partial^{2} \Pi}{\partial t_{1} \partial t_{2}}\left(\frac{f_{1} f_{2}}{A_{t_{1}}}, \frac{g_{1} g_{2}}{B_{t_{2}}}\right) \mathrm{d} t_{2} \mathrm{~d} t_{1} \\
= & \frac{1}{f_{1} f_{2} g_{1} g_{2}\left(f_{2}-f_{1}\right)\left(g_{2}-g_{1}\right)}\left[\prod\left(f_{1}, g_{2}\right)-\Gamma(\beta+1)\left(\frac{g_{1} g_{2}}{g_{2}-g_{1}}\right)^{\beta} J_{1 / g_{1}-}^{\beta}\left(\prod \circ \Omega_{2}\right)\left(f_{1}, \frac{1}{g_{2}}\right)\right. \\
& -\Gamma(\alpha+1)\left(\frac{f_{1} f_{2}}{f_{1}-f_{1}}\right)^{\alpha} J_{1 / f_{1}-}^{\alpha}\left(\prod \circ \Omega_{1}\right)\left(\frac{1}{f_{2}}, g_{1}\right)+\Gamma(\alpha+1) \Gamma(\beta+1) \\
& \left.\times\left(\frac{f_{1} f_{2}}{f_{1}-f_{1}}\right)^{\alpha}\left(\frac{g_{1} g_{2}}{g_{2}-g_{1}}\right)^{\beta} J_{1 / f_{1}-, 1 / g_{1}-}^{\alpha, \beta}\left(\prod \circ \Omega\right)\left(\frac{1}{f_{2}}, \frac{1}{g_{2}}\right)\right] .
\end{aligned}
$$

Thus from equalities (25)-(28), we have

$$
\begin{aligned}
I_{1}- & I_{2}-I_{3}+I_{4}=\frac{\prod\left(f_{2}, g_{2}\right)+\Pi\left(f_{1}, g_{1}\right)+\Pi\left(f_{2}, g_{1}\right)+\Pi\left(f_{1}, g_{2}\right)}{f_{1} f_{2} g_{1} g_{2}\left(f_{2}-f_{1}\right)\left(g_{2}-g_{1}\right)}-\frac{\Gamma(\beta+1)}{f_{1} f_{2} g_{1} g_{2}\left(f_{2}-f_{1}\right)\left(g_{2}-g_{1}\right)}\left(\frac{g_{1} g_{2}}{g_{2}-g_{1}}\right)^{\beta} \\
& \times\left[J_{1 / g_{2}+}^{\beta}\left(\prod \circ \Omega_{2}\right)\left(f_{2}, \frac{1}{g_{1}}\right)+J_{1 / g_{2}+}^{\beta}\left(\prod \circ \Omega_{2}\right)\left(f_{1}, \frac{1}{g_{1}}\right)+J_{1 / g_{1}-}^{\beta}\left(\prod \circ \Omega_{2}\right)\left(f_{2}, \frac{1}{g_{2}}\right)+J_{1 / g_{1}-}^{\beta}\left(\prod \circ \Omega_{2}\right)\right. \\
& \left.\times\left(f_{1}, \frac{1}{g_{2}}\right)\right]-\frac{\Gamma(\alpha+1)}{f_{1} f_{2} g_{1} g_{2}\left(f_{2}-f_{1}\right)\left(g_{2}-g_{1}\right)}\left(\frac{f_{1} f_{2}}{f_{2}-f_{1}}\right)^{\alpha}\left[J_{1 / f_{2}+}^{\alpha}\left(\prod \circ \Omega_{1}\right)\left(\frac{1}{f_{1}}, g_{2}\right)+J_{1 / f_{1}-}^{\alpha}\left(\prod \circ \Omega_{1}\right)\right. \\
& \left.\times\left(\frac{1}{f_{2}}, g_{2}\right)+J_{1 / f_{2}+}^{\alpha}\left(\prod \circ \Omega_{1}\right)\left(\frac{1}{f_{1}}, g_{1}\right)+J_{1 / f_{1}-}^{\alpha}\left(\prod \circ \Omega_{1}\right)\left(\frac{1}{f_{2}}, g_{1}\right)\right]+\frac{\Gamma(\alpha+1) \Gamma(\beta+1)}{f_{1} f_{2} g_{1} g_{2}\left(f_{2}-f_{1}\right)\left(g_{2}-g_{1}\right)} \\
& \times\left[J_{1 / f_{2}+, 1 / g_{2}+}^{\alpha, \beta}\left(\prod \circ \Omega\right)\left(\frac{1}{f_{1}}, \frac{1}{g_{1}}\right)+J_{1 / f_{1}-, 1 / g_{2}+}^{\alpha, \beta}\left(\prod \circ \Omega\right)\left(\frac{1}{f_{2}}, \frac{1}{g_{1}}\right)\right. \\
& \left.+J_{1 / f_{2}+, 1 / g_{1}-}^{\alpha, \beta}\left(\prod \circ \Omega\right)\left(\frac{1}{f_{1}}, \frac{1}{g_{2}}\right)+J_{1 / f_{1}-, 1 / g_{1}-}^{\alpha, \beta}\left(\prod \circ \Omega\right)\left(\frac{1}{f_{2}}, \frac{1}{g_{2}}\right)\right] .
\end{aligned}
$$

Multiplying both sides of equality (29) by $\frac{f_{1} f_{2} g_{1} g_{2}\left(f_{2}-f_{1}\right)\left(g_{2}-g_{1}\right)}{4}$, we get the desired equality (23).

Theorem 13. Let $\Pi: \Delta=\left[f_{1}, f_{2}\right] \times\left[g_{1}, g_{2}\right] \subseteq(0, \infty) \times(0, \infty) \rightarrow \mathbb{R}$ be a partial differentiable mapping on $\Delta$ with $0<f_{1}<f_{2}$ and $0<g_{1}<g_{2}$. If $\left|\partial^{2} \Pi / \partial t_{1} \partial t_{2}\right|$ is a harmonically convex on the co-ordinates on $\Delta$, then following holds:

$$
\begin{aligned}
& \mid \frac{\Pi\left(f_{1}, g_{1}\right)+\Pi\left(f_{1}, g_{2}\right)+\Pi\left(f_{2}, g_{1}\right)+\Pi\left(f_{2}, g_{2}\right)}{4}+\frac{\Gamma(\alpha+1) \Gamma(\beta+1)}{4}\left(\frac{f_{1} f_{2}}{f_{2}-f_{1}}\right)^{\alpha}\left(\frac{g_{1} g_{2}}{g_{2}-g_{1}}\right)^{\beta} \\
& \quad \times\left[J_{1 / f_{2}+, 1 / g_{1}+}^{\alpha, \beta}\left(\prod \circ \Omega\right)\left(\frac{1}{f_{1}}, \frac{1}{g_{1}}\right)+J_{1 / f_{1}-, 1 / g_{2}+}^{\alpha, \beta}\left(\prod \circ \Omega\right)\left(\frac{1}{f_{2}}, \frac{1}{g_{1}}\right)\right. \\
& \left.\quad+J_{1 / f_{2}+, 1 / g_{1}-}^{\alpha, \beta}\left(\prod \circ \Omega\right)\left(\frac{1}{f_{1}}, \frac{1}{g_{2}}\right)+J_{1 / f_{1}-, 1 / g_{1}-}^{\alpha, \beta}\left(\prod \circ \Omega\right)\left(\frac{1}{f_{2}}, \frac{1}{g_{2}}\right)\right]-\Xi \mid \\
& \leq \frac{f_{1} g_{1}\left(f_{2}-f_{1}\right)\left(g_{2}-g_{1}\right)}{4 f_{2} g_{2}(\alpha+1)(\beta+1)(\alpha+2)(\beta+2)}\left[\vartheta_{1}\left|\frac{\partial^{2} \Pi}{\partial t_{1} \partial t_{2}}\left(f_{1}, g_{1}\right)\right|+\vartheta_{2}\left|\frac{\partial^{2} \Pi}{\partial t_{1} \partial t_{2}}\left(f_{1}, g_{2}\right)\right|\right. \\
& \left.\quad+\vartheta_{3}\left|\frac{\partial^{2} \Pi}{\partial t_{1} \partial t_{2}}\left(f_{2}, g_{1}\right)\right|+\vartheta_{4}\left|\frac{\partial^{2} \Pi}{\partial t_{1} \partial t_{2}}\left(f_{2}, g_{2}\right)\right|\right]
\end{aligned}
$$


where

$$
\begin{aligned}
\vartheta_{1}= & (\alpha+1)(\beta+1){ }_{2} F_{1}\left(2, \alpha+2 ; \alpha+3 ; 1-\frac{f_{1}}{f_{2}}\right){ }_{2} F_{1}\left(2, \beta+2 ; \beta+3 ; 1-\frac{g_{1}}{g_{2}}\right) \\
& +(\beta+1){ }_{2} F_{1}\left(2,2 ; \alpha+3 ; 1-\frac{f_{1}}{f_{2}}\right){ }_{2} F_{1}\left(2, \beta+2 ; \beta+3 ; 1-\frac{g_{1}}{g_{2}}\right)+{ }_{2} F_{1}\left(2, \alpha+2 ; \alpha+3 ; 1-\frac{f_{1}}{f_{2}}\right) \\
& \times{ }_{2} F_{1}\left(2,2 ; \beta+3 ; 1-\frac{g_{1}}{g_{2}}\right)+{ }_{2} F_{1}\left(2,2 ; \alpha+3 ; 1-\frac{f_{1}}{f_{2}}\right){ }_{2} F_{1}\left(2,2 ; \beta+3 ; 1-\frac{g_{1}}{g_{2}}\right), \\
\vartheta_{2}= & (\beta+1){ }_{2} F_{1}\left(2, \alpha+1 ; \alpha+3 ; 1-\frac{f_{1}}{f_{2}}\right){ }_{2} F_{1}\left(2, \beta+2 ; \beta+3 ; 1-\frac{g_{1}}{g_{2}}\right) \\
& +(\alpha+1)(\beta+1){ }_{2} F_{1}\left(2,1 ; \alpha+3 ; 1-\frac{f_{1}}{f_{2}}\right){ }_{2} F_{1}\left(2, \beta+2 ; \beta+3 ; 1-\frac{g_{1}}{g_{2}}\right)+{ }_{2} F_{1}\left(2, \alpha+1 ; \alpha+3 ; 1-\frac{f_{1}}{f_{2}}\right) \\
& \times{ }_{2} F_{1}\left(2,2 ; \beta+3 ; 1-\frac{g_{1}}{g_{2}}\right)+{ }_{2} F_{1}\left(2,1 ; \alpha+3 ; 1-\frac{f_{1}}{f_{2}}\right){ }_{2} F_{1}\left(2,2 ; \beta+3 ; 1-\frac{g_{1}}{g_{2}}\right), \\
\vartheta_{3}= & (\alpha+1){ }_{2} F_{1}\left(2, \alpha+2 ; \alpha+3 ; 1-\frac{f_{1}}{f_{2}}\right){ }_{2} F_{1}\left(2, \beta+1 ; \beta+3 ; 1-\frac{g_{1}}{g_{2}}\right) \\
& +(\beta+1){ }_{2} F_{1}\left(2,2 ; \alpha+3 ; 1-\frac{f_{1}}{f_{2}}\right){ }_{2} F_{1}\left(2, \beta+1 ; \beta+3 ; 1-\frac{g_{1}}{g_{2}}\right)+(\beta+1){ }_{2} F_{1}\left(2, \alpha+2 ; \alpha+3 ; 1-\frac{f_{1}}{f_{2}}\right) \\
& \times{ }_{2} F_{1}\left(2,1 ; \beta+3 ; 1-\frac{g_{1}}{g_{2}}\right)+{ }_{2} F_{1}\left(2,2 ; \alpha+3 ; 1-\frac{f_{1}}{f_{2}}\right){ }_{2} F_{1}\left(2,1 ; \beta+3 ; 1-\frac{g_{1}}{g_{2}}\right), \\
\vartheta_{4}= & { }_{2} F_{1}\left(2, \alpha+1 ; \alpha+3 ; 1-\frac{f_{1}}{f_{2}}\right){ }_{2} F_{1}\left(2, \beta+1 ; \beta+3 ; 1-\frac{g_{1}}{g_{2}}\right) \\
& +(\alpha+1){ }_{2} F_{1}\left(2,1 ; \alpha+3 ; 1-\frac{f_{1}}{f_{2}}\right){ }_{2} F_{1}\left(2, \beta+1 ; \beta+3 ; 1-\frac{g_{1}}{g_{2}}\right)+(\beta+1){ }_{2} F_{1}\left(2, \alpha+1 ; \alpha+3 ; 1-\frac{f_{1}}{f_{2}}\right) \\
& \times{ }_{2} F_{1}\left(2,1 ; \beta+3 ; 1-\frac{g_{1}}{g_{2}}\right)+(\alpha+1)(\beta+1){ }_{2} F_{1}\left(2,1 ; \alpha+3 ; 1-\frac{f_{1}}{f_{2}}\right){ }_{2} F_{1}\left(2,1 ; \beta+3 ; 1-\frac{g_{1}}{g_{2}}\right) .
\end{aligned}
$$

Proof. Using Lemma 1, we have

$$
\begin{aligned}
& \frac{\prod\left(f_{1}, g_{1}\right)+\Pi\left(f_{1}, g_{2}\right)+\Pi\left(f_{2}, g_{1}\right)+\Pi\left(f_{2}, g_{2}\right)}{4}+\frac{\Gamma(\alpha+1) \Gamma(\beta+1)}{4}\left(\frac{f_{1} f_{2}}{f_{2}-f_{1}}\right)^{\alpha}\left(\frac{g_{1} g_{2}}{g_{2}-g_{1}}\right)^{\beta} \\
& \quad \times\left[J_{1 / f_{2}+, 1 / g_{1}+}^{\alpha, \beta}\left(\prod \circ \Omega\right)\left(\frac{1}{f_{1}}, \frac{1}{g_{1}}\right)+J_{1 / f_{1}-, 1 / g_{2}+}^{\alpha, \beta}\left(\prod \circ \Omega\right)\left(\frac{1}{f_{2}}, \frac{1}{g_{1}}\right)\right. \\
& \left.\quad+J_{1 / f_{2}+, 1 / g_{1}-}^{\alpha, \beta}\left(\prod \circ \Omega\right)\left(\frac{1}{f_{1}}, \frac{1}{g_{2}}\right)+J_{1 / f_{1}-, 1 / g_{1}-}^{\alpha, \beta}\left(\prod \circ \Omega\right)\left(\frac{1}{f_{2}}, \frac{1}{g_{2}}\right)\right]-\Xi \\
& =\frac{f_{1} f_{2} g_{1} g_{2}\left(f_{2}-f_{1}\right)\left(g_{2}-g_{1}\right)}{4}\left[\int_{0}^{1} \int_{0}^{1} \frac{r_{1}^{\alpha} r_{2}^{\beta}}{A_{t_{1}}^{2} B_{t_{2}}^{2}} \frac{\partial^{2} \Pi}{\partial t_{1} \partial t_{2}}\left(\frac{f_{1} f_{2}}{A_{t_{1}}}, \frac{g_{1} g_{2}}{B_{t_{2}}}\right) \mathrm{d} t_{2} \mathrm{~d} t_{1}\right. \\
& \quad+\int_{0}^{1} \int_{0}^{1} \frac{\left(1-t_{1}\right)^{\alpha} t_{2}^{\beta}}{A_{t_{1}}^{2} B_{t_{2}}^{2}} \frac{\partial^{2} \Pi}{\partial t_{1} \partial t_{2}}\left(\frac{f_{1} f_{2}}{A_{t_{1}}}, \frac{g_{1} g_{2}}{B_{t_{2}}}\right) \mathrm{d} t_{2} \mathrm{~d} t_{1}+\int_{0}^{1} \int_{0}^{1} \frac{t_{1}^{\alpha}\left(1-t_{2}\right)^{\beta}}{A_{t_{1}}^{2} B_{t_{2}}^{2}} \frac{\partial^{2} \Pi}{\partial t_{1} \partial t_{2}}\left(\frac{f_{1} f_{2}}{A_{t_{1}}}, \frac{g_{1} g_{2}}{B_{t_{2}}}\right) \mathrm{d} t_{2} \mathrm{~d} t_{1} \\
& \left.\quad+\int_{0}^{1} \int_{0}^{1} \frac{\left(1-t_{1}\right)^{\alpha}\left(1-t_{2}\right)^{\beta}}{A_{t_{1}}^{2} B_{t_{2}}^{2}} \frac{\partial^{2} \Pi}{\partial t_{1} \partial t_{2}}\left(\frac{f_{1} f_{2}}{A_{t_{1}}}, \frac{g_{1} g_{2}}{B_{t_{2}}}\right) \mathrm{d} t_{2} \mathrm{~d} t_{1}\right] .
\end{aligned}
$$

Now using co-ordinated harmonically convexity of $\left|\frac{\partial^{2} \Pi}{\partial t_{1} \partial t_{2}}\right|$, we get 


$$
\begin{aligned}
& \mid \frac{\prod\left(f_{1}, g_{1}\right)+\Pi\left(f_{1}, g_{2}\right)+\Pi\left(f_{2}, g_{1}\right)+\Pi\left(f_{2}, g_{2}\right)}{4}+\frac{\Gamma(\alpha+1) \Gamma(\beta+1)}{4}\left(\frac{f_{1} f_{2}}{f_{2}-f_{1}}\right)^{\alpha}\left(\frac{g_{1} g_{2}}{g_{2}-g_{1}}\right)^{\beta} \\
& \times\left[J_{1 / f_{2}+, 1 / g_{1}+}^{\alpha, \beta}\left(\prod \circ \Omega\right)\left(\frac{1}{f_{1}}, \frac{1}{g_{1}}\right)+J_{1 / f_{1}-, 1 / g_{2}+}^{\alpha, \beta}\left(\prod \circ \Omega\right)\left(\frac{1}{f_{2}}, \frac{1}{g_{1}}\right)\right. \\
& \left.+J_{1 / f_{2}+, 1 / g_{1}-}^{\alpha, \beta}\left(\prod \circ \Omega\right)\left(\frac{1}{f_{1}}, \frac{1}{g_{2}}\right)+J_{1 / f_{1}-, 1 / g_{1}-}^{\alpha, \beta}\left(\prod \circ \Omega\right)\left(\frac{1}{f_{2}}, \frac{1}{g_{2}}\right)\right]-\Xi \mid \\
& \leq \frac{f_{1} f_{2} g_{1} g_{2}\left(f_{2}-f_{1}\right)\left(g_{2}-g_{1}\right)}{4}\left[\int _ { 0 } ^ { 1 } \int _ { 0 } ^ { 1 } \left\{\frac{t_{1}^{\alpha} t_{2}^{\beta}}{A_{t_{1}}^{2} B_{t_{2}}^{2}}+\frac{\left(1-t_{1}\right)^{\alpha} t_{2}^{\beta}}{A_{t_{1}}^{2} B_{t_{2}}^{2}}+\frac{t_{1}^{\alpha}\left(1-t_{2}\right)^{\beta}}{A_{t_{1}}^{2} B_{t_{2}}^{2}}\right.\right. \\
& \left.+\frac{\left(1-t_{1}\right)^{\alpha}\left(1-t_{2}\right)^{\beta}}{A_{t_{1}}^{2} B_{t_{2}}^{2}}\right\}\left\{t_{1} t_{2}\left|\frac{\partial^{2} \Pi}{\partial t_{1} \partial t_{2}}\left(f_{1}, g_{1}\right)\right|+\left(1-t_{1}\right) t_{2}\left|\frac{\partial^{2} \Pi}{\partial t_{1} \partial t_{2}}\left(f_{2}, g_{1}\right)\right|\right. \\
& \left.\left.+t_{1}\left(1-t_{2}\right)\left|\frac{\partial^{2} \Pi}{\partial t_{1} \partial t_{2}}\left(f_{1}, g_{2}\right)\right|+\left(1-t_{1}\right)\left(1-t_{2}\right)\left|\frac{\partial^{2} \Pi}{\partial t_{1} \partial t_{2}}\left(f_{2}, g_{2}\right)\right|\right\} \mathrm{d} t_{2} \mathrm{~d} t_{1}\right] \\
& =\frac{f_{1} f_{2} g_{1} g_{2}\left(f_{2}-f_{1}\right)\left(g_{2}-g_{1}\right)}{4}\left[\int_{0}^{1} \int_{0}^{1} t_{1} t_{2}\left\{\frac{t_{1}^{\alpha} t_{2}^{\beta}}{A_{t_{1}}^{2} B_{t_{2}}^{2}}+\frac{\left(1-t_{1}\right)^{\alpha} t_{2}^{\beta}}{A_{t_{1}}^{2} B_{t_{2}}^{2}}+\frac{t_{1}^{\alpha}\left(1-t_{2}\right)^{\beta}}{A_{t_{1}}^{2} B_{t_{2}}^{2}}+\frac{\left(1-t_{1}\right)^{\alpha}\left(1-t_{2}\right)^{\beta}}{A_{t_{1}}^{2} B_{t_{2}}^{2}}\right\}\right. \\
& \times\left|\frac{\partial^{2} \Pi}{\partial t_{1} \partial t_{2}}\left(f_{1}, g_{1}\right)\right| \mathrm{d} t_{1} \mathrm{~d} t_{2}+\int_{0}^{1} \int_{0}^{1}\left(1-t_{1}\right) t_{2}\left\{\frac{t_{1}^{\alpha} t_{2}^{\beta}}{A_{t_{1}}^{2} B_{t_{2}}^{2}}+\frac{\left(1-t_{1}\right)^{\alpha} t_{2}^{\beta}}{A_{t_{1}}^{2} B_{t_{2}}^{2}}+\frac{t_{1}^{\alpha}\left(1-t_{2}\right)^{\beta}}{A_{t_{1}}^{2} B_{t_{2}}^{2}}+\frac{\left(1-t_{1}\right)^{\alpha}\left(1-t_{2}\right)^{\beta}}{A_{t_{1}}^{2} B_{t_{2}}^{2}}\right\} \\
& \times\left|\frac{\partial^{2} \Pi}{\partial t_{1} \partial t_{2}}\left(f_{2}, g_{1}\right)\right| \mathrm{d} t_{1} \mathrm{~d} t_{2}+\int_{0}^{1} \int_{0}^{1} t_{1}\left(1-t_{2}\right)\left\{\frac{t_{1}^{\alpha} t_{2}^{\beta}}{A_{t_{1}}^{2} B_{t_{2}}^{2}}+\frac{\left(1-t_{1}\right)^{\alpha} t_{2}^{\beta}}{A_{t_{1}}^{2} B_{t_{2}}^{2}}+\frac{t_{1}^{\alpha}\left(1-t_{2}\right)^{\beta}}{A_{t_{1}}^{2} B_{t_{2}}^{2}}+\frac{\left(1-t_{1}\right)^{\alpha}\left(1-t_{2}\right)^{\beta}}{A_{t_{1}}^{2} B_{t_{2}}^{2}}\right\} \\
& \times\left|\frac{\partial^{2} \Pi}{\partial t_{1} \partial t_{2}}\left(f_{1}, g_{2}\right)\right| \mathrm{d} t_{1} \mathrm{~d} t_{2}+\int_{0}^{1} \int_{0}^{1}\left(1-t_{1}\right)\left(1-t_{2}\right) \\
& \left.\times\left\{\frac{t_{1}^{\alpha} t_{2}^{\beta}}{A_{t_{1}}^{2} B_{t_{2}}^{2}}+\frac{\left(1-t_{1}\right)^{\alpha} t_{2}^{\beta}}{A_{t_{1}}^{2} B_{t_{2}}^{2}}+\frac{t_{1}^{\alpha}\left(1-t_{2}\right)^{\beta}}{A_{t_{1}}^{2} B_{t_{2}}^{2}}+\frac{\left(1-t_{1}\right)^{\alpha}\left(1-t_{2}\right)^{\beta}}{A_{t_{1}}^{2} B_{t_{2}}^{2}}\right\}\left|\frac{\partial^{2} \Pi}{\partial t_{1} \partial t_{2}}\left(f_{2}, g_{2}\right)\right| \mathrm{d} t_{1} \mathrm{~d} t_{2}\right] .
\end{aligned}
$$

After calculating above integrations, we get the required result.

Theorem 14. Let $\Pi: \Delta=\left[f_{1}, f_{2}\right] \times\left[g_{1}, g_{2}\right] \subseteq(0, \infty) \times(0, \infty) \rightarrow \mathbb{R}$ be a partial differentiable mapping on $\Delta$ with $0<f_{1}<f_{2}$ and $0<g_{1}<g_{2}$. If $\left|\partial^{2} \Pi / \partial t_{1} \partial t_{2}\right|^{q}, q>1$, is a harmonically convex on the co-ordinates on $\Delta$, then following holds:

$$
\begin{aligned}
& \mid \frac{\prod\left(f_{1}, g_{1}\right)+\Pi\left(f_{1}, g_{2}\right)+\Pi\left(f_{2}, g_{1}\right)+\Pi\left(f_{2}, g_{2}\right)}{4}+\frac{\Gamma(\alpha+1) \Gamma(\beta+1)}{4}\left(\frac{f_{1} f_{2}}{f_{2}-f_{1}}\right)^{\alpha}\left(\frac{g_{1} g_{2}}{g_{2}-g_{1}}\right)^{\beta} \\
& \quad \times\left[J_{1 / f_{2}+, 1 / g_{1}+}^{\alpha, \beta}\left(\prod \circ \Omega\right)\left(\frac{1}{f_{1}}, \frac{1}{g_{1}}\right)+J_{1 / f_{1}-, 1 / g_{2}+}^{\alpha, \beta}\left(\prod \circ \Omega\right)\left(\frac{1}{f_{2}}, \frac{1}{g_{1}}\right)\right. \\
& \left.\quad+J_{1 / f_{2}+, 1 / g_{1}-}^{\alpha, \beta}\left(\prod \circ \Omega\right)\left(\frac{1}{f_{1}}, \frac{1}{g_{2}}\right)+J_{1 / f_{1}-, 1 / g_{1}-}^{\alpha, \beta}\left(\prod \circ \Omega\right)\left(\frac{1}{f_{2}}, \frac{1}{g_{2}}\right)\right]-\Xi \mid \\
& \leq \frac{f_{1} g_{1}\left(f_{2}-f_{1}\right)\left(g_{2}-g_{1}\right)}{4 f_{2} g_{2}[(p \alpha+1)(p \beta+1)]^{1 / p}}\left[\psi_{1}^{1 / p}+\psi_{2}^{1 / p}+\psi_{3}^{1 / p}+\psi_{4}^{1 / p}\right] \\
& \quad \times\left(\frac{\left|\frac{\partial^{2} \Pi}{\partial t_{1} \partial t_{2}}\left(f_{1}, g_{1}\right)\right|^{q}+\left|\frac{\partial^{2} \Pi}{\partial t_{1} \partial t_{2}}\left(f_{1}, g_{2}\right)\right|^{q}+\left|\frac{\partial^{2} \Pi}{\partial t_{1} \partial t_{2}}\left(f_{2}, g_{1}\right)\right|^{q}+\left|\frac{\partial^{2} \Pi}{\partial t_{1} \partial t_{2}}\left(f_{2}, g_{2}\right)\right|^{q}}{4}\right)^{1 / q},
\end{aligned}
$$

where

$$
\psi_{1}={ }_{2} F_{1}\left(2 p, p \alpha+1 ; p \alpha+2 ; 1-\frac{f_{1}}{f_{2}}\right){ }_{2} F_{1}\left(2 p, p \beta+1 ; p \beta+2 ; 1-\frac{g_{1}}{g_{2}}\right)
$$




$$
\begin{aligned}
& \psi_{2}={ }_{2} F_{1}\left(2 p, 1 ; p \alpha+2 ; 1-\frac{f_{1}}{f_{2}}\right){ }_{2} F_{1}\left(2 p, p \beta+1 ; p \beta+2 ; 1-\frac{g_{1}}{g_{2}}\right), \\
& \psi_{3}={ }_{2} F_{1}\left(2 p, p \alpha+1 ; p \alpha+2 ; 1-\frac{f_{1}}{f_{2}}\right){ }_{2} F_{1}\left(2 p, 1 ; p \beta+2 ; 1-\frac{g_{1}}{g_{2}}\right), \\
& \psi_{4}={ }_{2} F_{1}\left(2 p, 1 ; p \alpha+2 ; 1-\frac{f_{1}}{f_{2}}\right){ }_{2} F_{1}\left(2 p, 1 ; p \beta+2 ; 1-\frac{g_{1}}{g_{2}}\right) .
\end{aligned}
$$

Proof. Applying the Holder's inequality for double integrals in (35), we get

$$
\begin{aligned}
& \mid \frac{\prod\left(f_{1}, g_{1}\right)+\Pi\left(f_{1}, g_{2}\right)+\Pi\left(f_{2}, g_{1}\right)+\Pi\left(f_{2}, g_{2}\right)}{4}+\frac{\Gamma(\alpha+1) \Gamma(\beta+1)}{4}\left(\frac{f_{1} f_{2}}{f_{2}-f_{1}}\right)^{\alpha}\left(\frac{g_{1} g_{2}}{g_{2}-g_{1}}\right)^{\beta} \\
& \quad \times\left[J_{1 / f_{2}+, 1 / g_{1}+}^{\alpha, \beta}\left(\prod \circ \Omega\right)\left(\frac{1}{f_{1}}, \frac{1}{g_{1}}\right)+J_{1 / f_{1}-, 1 / g_{2}+}^{\alpha, \beta}\left(\prod \circ \Omega\right)\left(\frac{1}{f_{2}}, \frac{1}{g_{1}}\right)\right. \\
& \left.\quad+J_{1 / f_{2}+, 1 / g_{1}-}^{\alpha, \beta}\left(\prod \circ \Omega\right)\left(\frac{1}{f_{1}}, \frac{1}{g_{2}}\right)+J_{1 / f_{1}-, 1 / g_{1}-}^{\alpha, \beta}\left(\prod \circ \Omega\right)\left(\frac{1}{f_{2}}, \frac{1}{g_{2}}\right)\right]-\Xi \mid \\
& \leq \frac{f_{1} f_{2} g_{1} g_{2}\left(f_{2}-f_{1}\right)\left(g_{2}-g_{1}\right)}{4}\left[\left(\int_{0}^{1} \int_{0}^{1} \frac{t_{1}^{p \alpha} t_{2}^{p \beta}}{A_{t_{1}}^{2 p} B_{t_{2}}^{2 p}} d t_{2} d t_{1}\right)^{1 / p}+\left(\int_{0}^{1} \int_{0}^{1} \frac{\left(1-t_{1}\right)^{p \alpha} t_{2}^{p \beta}}{A_{t_{1}}^{2 p} B_{t_{2}}^{2 p}} d t_{2} d t_{1}\right)^{1 / p}\right. \\
& \left.\quad+\left(\int_{0}^{1} \int_{0}^{1} \frac{t_{1}^{p \alpha}\left(1-t_{2}\right)^{p \beta}}{A_{t_{1}}^{2 p} B_{t_{2}}^{2 p}} d t_{2} d t_{1}\right)^{1 / p}+\left(\int_{0}^{1} \int_{0}^{1} \frac{\left(1-t_{1}\right)^{p \alpha}\left(1-t_{2}\right)^{p \beta}}{A_{t_{1}}^{2 p} B_{t_{2}}^{2 p}} d t_{2} d t_{1}\right)^{1 / p}\right] \\
& \quad \times\left(\int_{0}^{1} \int_{0}^{1}\left|\frac{\partial^{2} \Pi}{\partial t_{1} \partial t_{2}}\left(\frac{f_{1} f_{2}}{A_{t_{1}}}, \frac{g_{1} g_{2}}{B_{t_{2}}}\right)\right|^{q} d t_{1} d t_{2}\right)^{1 / q} .
\end{aligned}
$$

Using co-ordinated harmonically convexity of $\left|\frac{\partial^{2} \Pi}{\partial t_{1} \partial t_{2}}\right|^{q}$, we get

$$
\begin{aligned}
& \mid \frac{\Pi\left(f_{1}, g_{1}\right)+\Pi\left(f_{1}, g_{2}\right)+\Pi\left(f_{2}, g_{1}\right)+\Pi\left(f_{2}, g_{2}\right)}{4}+\frac{\Gamma(\alpha+1) \Gamma(\beta+1)}{4}\left(\frac{f_{1} f_{2}}{f_{2}-f_{1}}\right)^{\alpha}\left(\frac{g_{1} g_{2}}{g_{2}-g_{1}}\right)^{\beta} \\
& \times\left[J_{1 / f_{2}+, 1 / g_{1}+}^{\alpha, \beta}\left(\prod \circ \Omega\right)\left(\frac{1}{f_{1}}, \frac{1}{g_{1}}\right)+J_{1 / f_{1}-, 1 / g_{2}+}^{\alpha, \beta}\left(\prod \circ \Omega\right)\left(\frac{1}{f_{2}}, \frac{1}{g_{1}}\right)\right. \\
& \left.+J_{1 / f_{2}+, 1 / g_{1}-}^{\alpha, \beta}\left(\prod \circ \Omega\right)\left(\frac{1}{f_{1}}, \frac{1}{g_{2}}\right)+J_{1 / f_{1}-, 1 / g_{1}-}^{\alpha, \beta}\left(\prod \circ \Omega\right)\left(\frac{1}{f_{2}}, \frac{1}{g_{2}}\right)\right]-\Xi \mid \\
& \leq \frac{f_{1} f_{2} g_{1} g_{2}\left(f_{2}-f_{1}\right)\left(g_{2}-g_{1}\right)}{4}\left[\left(\int_{0}^{1} \int_{0}^{1} \frac{t_{1}^{p \alpha} t_{2}^{p \beta}}{A_{t_{1}}^{2 p} B_{t_{2}}^{2 p}} d t_{2} d t_{1}\right)^{1 / p}+\left(\int_{0}^{1} \int_{0}^{1} \frac{\left(1-t_{1}\right)^{p \alpha} t_{2}^{p \beta}}{A_{t_{1}}^{2 p} B_{t_{2}}^{2 p}} d t_{2} d t_{1}\right)^{1 / p}\right. \\
& \left.+\left(\int_{0}^{1} \int_{0}^{1} \frac{t_{1}^{p \alpha}\left(1-t_{2}\right)^{p \beta}}{A_{t_{1}}^{2 p} B_{t_{2}}^{2 p}} d t_{2} d t_{1}\right)^{1 / p}+\left(\int_{0}^{1} \int_{0}^{1} \frac{\left(1-t_{1}\right)^{p \alpha}\left(1-t_{2}\right)^{p \beta}}{A_{t_{1}}^{2 p} B_{t_{2}}^{2 p}} d t_{2} d t_{1}\right)^{1 / p}\right] \\
& \times\left(\int _ { 0 } ^ { 1 } \int _ { 0 } ^ { 1 } \left\{t_{1} t_{2}\left|\frac{\partial^{2} \Pi}{\partial t_{1} \partial t_{2}}\left(f_{1}, f_{2}\right)\right|^{q}+\left(1-t_{1}\right) t_{2}\left|\frac{\partial^{2} \Pi}{\partial t_{1} \partial t_{2}}\left(f_{2}, g_{1}\right)\right|^{q}\right.\right. \\
& \left.\left.+t_{1}\left(1-t_{2}\right)\left|\frac{\partial^{2} \Pi}{\partial t_{1} \partial t_{2}}\left(f_{1}, g_{2}\right)\right|^{q}+\left(1-t_{1}\right)\left(1-t_{2}\right)\left|\frac{\partial^{2} \Pi}{\partial t_{1} \partial t_{2}}\left(f_{2}, g_{2}\right)\right|^{q}\right\} d t_{2} d t_{1}\right)^{1 / q} \\
& =\frac{f_{1} f_{2} g_{1} g_{2}\left(f_{2}-f_{1}\right)\left(g_{2}-g_{1}\right)}{4}\left[\left(\int_{0}^{1} \int_{0}^{1} \frac{t_{1}^{p \alpha} t_{2}^{p \beta}}{A_{t_{1}}^{2 p} B_{t_{2}}^{2 p}} d t_{2} d t_{1}\right)^{1 / p}+\left(\int_{0}^{1} \int_{0}^{1} \frac{\left(1-t_{1}\right)^{p \alpha} t_{2}^{p \beta}}{A_{t_{1}}^{2 p} B_{t_{2}}^{2 p}} d t_{2} d t_{1}\right)^{1 / p}\right.
\end{aligned}
$$




$$
\begin{aligned}
& \left.+\left(\int_{0}^{1} \int_{0}^{1} \frac{t_{1}^{p \alpha}\left(1-t_{2}\right)^{p \beta}}{A_{t_{1}}^{2 p} B_{t_{2}}^{2 p}} d t_{2} d t_{1}\right)^{1 / p}+\left(\int_{0}^{1} \int_{0}^{1} \frac{\left(1-t_{1}\right)^{p \alpha}\left(1-t_{2}\right)^{p \beta}}{A_{t_{1}}^{2 p} B_{t_{2}}^{2 p}} d t_{2} d t_{1}\right)^{1 / p}\right] \\
& \times\left(\frac{\left|\frac{\partial^{2} \Pi}{\partial t_{1} \partial t_{2}}\left(f_{1}, g_{1}\right)\right|^{q}+\left|\frac{\partial^{2} \Pi}{\partial t_{1} \partial t_{2}}\left(f_{1}, g_{2}\right)\right|^{q}+\left|\frac{\partial^{2} \Pi}{\partial t_{1} \partial t_{2}}\left(f_{2}, g_{1}\right)\right|^{q}+\left|\frac{\partial^{2} \Pi}{\partial t_{1} \partial t_{2}}\left(f_{2}, g_{2}\right)\right|^{q}}{4}\right)^{1 / q}
\end{aligned}
$$

By calculating all integrals, we get the required result (37).

\section{Conclusion}

In Theorem 11 and 12, we have proved some new Hermite-Hadamard type inequalities for co-ordinated harmonically convex on a rectangle via Riemann-Liouville fractional integrals. In Lemma 1, we have proved a fractional integral identity and then with the help of this Lemma 1 we proved some fractional Hermite-Hadamard type inequalities on the co-ordinates.

Acknowledgments: The present investigation is supported by National University of Science and Technology(NUST), Islamabad, Pakistan.

Author Contributions: All authors contributed equally to the writing of this paper. All authors read and approved the final manuscript.

Conflicts of Interest: "The authors declare no conflict of interest."

\section{References}

[1] Hadamard, J. (1893). Étude sur les propriétés des fonctions entières et en particulier d'une fonction considérée par Riemann. Journal de Mathématiques Pures et Appliquées, 58, 171-216.

[2] Mehreen, N., \& Anwar, M. (2020). Hermite-Hadamard type inequalities via exponentially $(p, h)$-convex functions. IEEE Access, 8, 37589-37595.

[3] Mehreen, N., \& Anwar, M. (2020). Hermite-Hadamard and Hermite-Hadamard-Fejér type inequalities for $p$-convex functions via conformable fractional integrals. Journal of Inequalities and Applications, 2020, Article No. 107.

[4] Mehreen, N., \& Anwar, M. (2019). Hermite-Hadamard and Hermite-Hadamard-Fejér type inequalities for $p$-convex functions via new fractional conformable integral operators. Journal of Mathematics and Computer Science, 19(04), 230-240.

[5] Mehreen, N., \& Anwar, M. (2019). Hermite-Hadamard type inequalities for exponentially $p$-convex functions and exponentially s-convex functions in the second sense with applications. Journal of Inequalities and Applications, 2019, Article No. 92.

[6] Mehreen, N., \& Anwar, M. (2018). Integral inequalities for some convex functions via generalized fractional integrals. Journal of inequalities and applications, 2018, Article No. 208.

[7] Mehreen, N., \& Anwar, M. (2019). Some inequalities via $\phi$-RiemannÜLiouville fractional integrals. AIMS Mathematics, 4(5), 1403-1415.

[8] Mehreen, N., \& Anwar, M. (2020). On some Hermite-Hadamard type inequalities for tgs tgs-convex functions via generalized fractional integrals. Advances in Difference Equations, 2020, Article No. 6.

[9] Noor, M. A., Noor, K. I., Iftikhar, S., \& Ionescu, C. (2017). Hermite-Hadamard inequalities for co-ordinated harmonic convex functions. University Politehnica of Bucharest Scientific Bulletin-Series A-Applied Mathematics and Physics, 79, $25-34$.

[10] Dragomir, S. S. (2001). On the Hadamard's inequlality for convex functions on the co-ordinates in a rectangle from the plane. Taiwanese Journal of Mathematics, 5(4) 775-788.

[11] Sarikaya, M. Z. (2014). On the Hermite-Hadamard-type inequalities for co-ordinated convex function via fractional integrals. Integral Transforms and Special Functions, 25(2), 134-147.

[12] Noor, M. A., Noor, K. I., \& Awan, M. U. (2015). Integral inequalities for coordinated harmonically convex functions. Complex Variables and Elliptic Equations, 60(6), 776-786.

[13] Kilbas, A. A., Srivastava, H. M., \& Trujillo, J. J. (2006). Theory and Applications of Fractional Differential Equations (Vol. 204). elsevier.

[14] Iscan, I., \& Wu, S. (2014). Hermite-Hadamard type inequalities for harmonically convex functions via fractional integrals. Applied Mathematics and Computation, 238, 237-244. 
(C) 2020 by the authors; licensee PSRP, Lahore, Pakistan. This article is an open access article distributed under the terms and conditions of the Creative Commons Attribution (CC-BY) license (http://creativecommons.org/licenses/by/4.0/). 